\title{
BIERI-NEUMANN-STREBEL-RENZ INVARIANTS AND HOMOLOGY JUMPING LOCI
}

\author{
STEFAN PAPADIMA ${ }^{1}$ AND ALEXANDER I. SUCIU ${ }^{2}$
}

\begin{abstract}
We investigate the relationship between the geometric Bieri-NeumannStrebel-Renz invariants of a space (or of a group), and the jump loci for homology with coefficients in rank 1 local systems over a field. We give computable upper bounds for the geometric invariants, in terms of the exponential tangent cones to the jump loci over the complex numbers. Under suitable hypotheses, these bounds can be expressed in terms of simpler data, for instance, the resonance varieties associated to the cohomology ring. These techniques yield information on the homological finiteness properties of free abelian covers of a given space, and of normal subgroups with abelian quotients of a given group. We illustrate our results in a variety of geometric and topological contexts, such as toric complexes and Artin kernels, as well as Kähler and quasi-Kähler manifolds.
\end{abstract}

\section{Contents}

1. Introduction 2

2. Homological finiteness properties $\quad 7$

3. Characteristic varieties and Alexander varieties 8

4. Tangent cones and exponential tangent cones 11

5. Resonance varieties, formality, and the exponential formula 13

6. Homology of free abelian covers 15

7. Bieri-Neumann-Strebel-Renz invariants 17

8. Novikov homology 19

9. $\quad \Sigma$-invariants and jumping loci over $\mathbb{C} \quad 21$

10. $\Sigma$-invariants and jumping loci in arbitrary characteristic 23

11. $\Sigma$-invariants, 1-formality, and deficiency 24

12. Finitely generated nilpotent groups 26

13. Products and coproducts 27

14. Toric complexes and right-angled Artin groups 29

15. Free abelian covers of toric complexes and Artin kernels 31

16. Kähler and quasi-Kähler manifolds 35

References $\quad 38$

2000 Mathematics Subject Classification. Primary 20J05, 55N25. Secondary 14F35, 20F36, 20 F65.

Key words and phrases. Characteristic variety, Alexander variety, resonance variety, exponential tangent cone, homology of free abelian covers, Bieri-Neumann-Strebel-Renz invariant, Novikov homology, valuation, algebraic integer, right-angled Artin group, Artin kernel, Kähler manifold, quasi-Kähler manifold.

${ }^{1}$ Partially supported by grant CNCSIS ID-1189/2009-2011 of the Romanian Ministry of Education and Research.

${ }^{2}$ Partially supported by NSA grant H98230-09-1-0012, and an ENHANCE grant from Northeastern University. 


\section{INTRODUCTION}

1.1. $\Sigma$-invariants of groups and spaces. In their landmark paper [10], Bieri, Neumann, and Strebel associated to every finitely generated group $G$ an open, conical subset $\Sigma^{1}(G)$ of the real vector space $\operatorname{Hom}(G, \mathbb{R})$. The BNS invariant and its higher-order generalizations, the invariants $\Sigma^{r}(G, \mathbb{Z})$ of Bieri and Renz [11], hold subtle information about the homological finiteness properties of normal subgroups of $G$ with abelian quotients. The actual computation of the $\Sigma$-invariants is enormously complicated, and has been achieved so far only for some special classes of groups, such as metabelian groups [9], one-relator groups [12], and right-angled Artin groups [39].

The definition of the Bieri-Neumann-Strebel-Renz invariants was further extended to connected, finite CW-complexes by Farber, Geoghegan, and Schütz [28]. Slightly more generally, let $X$ be a connected CW-complex with finite 1-skeleton, and fundamental group $G$. Based on a key result of Sikorav [50], we will say that a nontrivial character $\chi \in$ $\operatorname{Hom}(G, \mathbb{R})$ belongs to $\Sigma^{q}(X, \mathbb{Z})$, for $q \geq 0$, if the twisted homology of $X$ with coefficients in the Novikov-Sikorav completion of the group ring, $\widehat{\mathbb{Z} G}_{-\chi}$, vanishes up to degree $q$. If the group $G$ is of type $\mathrm{F}_{k}$, i.e., admits a classifying space $K(G, 1)$ with finite $k$-skeleton, then $\Sigma^{q}(G, \mathbb{Z})=\Sigma^{q}(K(G, 1), \mathbb{Z})$, for all $q \leq k$. See $\S \S 8.1-8.2$ for more details.

In this paper, we prove that each BNSR invariant $\Sigma^{q}(X, \mathbb{Z})$ is contained in the complement of a rationally defined subspace arrangement in the vector space $\operatorname{Hom}\left(\pi_{1}(X), \mathbb{R}\right)$. Our upper bounds for the $\Sigma$-invariants of spaces and groups are effectively computable, and, in many cases, sharp. The bounds are derived from the characteristic varieties, defined as the jump loci for the homology of $X$ with coefficients in rank 1 local systems. Under a certain hypothesis, these upper bounds can be expressed in terms of even simpler data, namely, the resonance varieties associated to the cohomology ring of $X$.

To obtain our results, we make essential use of the exponential tangent cone construction, introduced in our joint work with A. Dimca [23]. Another important ingredient is the relationship between the characteristic varieties, their (exponential) tangent cones at the origin, and the resonance varieties, also developed in [23].

1.2. Fibrations, Alexander polynomial, and Thurston norm. Before describing our work in more detail, let us briefly review some of the topological context, starting with the following question: When does a compact, connected manifold $M^{n}$ fiber over the circle? In dimension 3, Stallings [51] reduced this question to a purely group-theoretical one: If $\pi_{1}\left(M^{3}\right)$ admits a surjective homomorphism to $\mathbb{Z}$ with finitely generated kernel $N$, then $M^{3}$ fibers over $S^{1}$, with the fiber having fundamental group $N$.

This striking result led to a follow-up question: Given a finitely generated group $G$, and an epimorphism $\nu: G \rightarrow \mathbb{Z}$, what are the finiteness properties of $N=\operatorname{ker}(\nu)$ ? It was Stallings again who showed in [52] that both $G$ and $N$ could be finitely presented, and yet $H_{3}(N, \mathbb{Z})$ fails to be finitely generated; in particular, such $N$ is not of type $\mathrm{FP}_{3}$. (The finiteness properties of spaces and groups are reviewed in $\S 2$.)

An old observation in knot theory is that the Alexander polynomial $\Delta_{K}(t)$ of a fibered knot $K$ is monic. More generally, Dwyer and Freed [25] showed that the support varieties of the Alexander invariants of a finite CW-complex completely determine the homological finiteness properties of its free abelian covers. 
For each compact, orientable 3-manifold $M$, Thurston [54] defined a (pseudo-)norm on $H^{1}(M, \mathbb{R})$, which measures the minimal complexity of an embedded surface dual to a given cohomology class. In [10], Bieri, Neumann, and Strebel found a remarkable connection between the Thurston norm of $M$ and the BNS invariant of $G=\pi_{1}(M)$, thereby recasting Stallings' fibration theorem in a more general framework.

1.3. Homology jumping loci and tangent cones. We now return to one of the central objects of our study. Let $X$ be a connected CW-complex with finitely many cells up to dimension $k$, for some $k \geq 1$. Let $G=\pi_{1}(X)$, and fix a coefficient field $\mathbb{k}$. Each character $\rho: G \rightarrow \mathbb{k}^{\times}$gives rise to a rank 1 local system on $X$, call it $\mathbb{k}_{\rho}$. The characteristic varieties of $X$ are Zariski closed subsets of the algebraic group $\operatorname{Hom}\left(G, \mathbb{k}^{\times}\right)$, defined as follows. For each $0 \leq i \leq k$ and $d>0$, the variety $\mathcal{V}_{d}^{i}(X, \mathbb{k})$ consists of those characters $\rho$ for which the dimension of $H_{i}\left(X, \mathbb{k}_{\rho}\right)$ is at least $d$. As we show in $\S 3$, these varieties are closely related to the support loci of the elementary ideals of the Alexander invariants $H_{i}\left(X^{\mathrm{ab}}, \mathbb{k}\right)$, where $X^{\mathrm{ab}}$ is the maximal abelian cover of $X$.

In $\S 4$, we discuss two types of approximations to the variety $W=\mathcal{V}_{d}^{i}(X, \mathbb{C})$ around the trivial character $1 \in \operatorname{Hom}\left(G, \mathbb{C}^{\times}\right)$. One is the usual tangent cone, $T C_{1}(W)$, while the other is the exponential tangent cone, $\tau_{1}(W)$, which consists of those classes $z \in H^{1}(X, \mathbb{C})$ for which the curve $\{\exp (t z) \mid t \in \mathbb{C}\}$ is completely contained in $W$. It turns out that $\tau_{1}(W)$ is a finite union of rationally defined linear subspaces, all contained in $T C_{1}(W)$. In general, this inclusion is strict, but, if all irreducible components of $W$ containing 1 are subtori, then $\tau_{1}(W)=T C_{1}(W)$.

We turn in $\S 5$ to another type of jumping loci, depending only on the cohomology algebra $A=H^{*}(X, \mathbb{k})$. If char $\mathbb{k}=2$, assume $H_{1}(X, \mathbb{Z})$ has no 2-torsion. Then, for each $z \in A^{1}$, right-multiplication by $z$ defines a cochain complex $(A, z)$. The resonance varieties of $X$ are homogeneous subvarieties of the affine space $\operatorname{Hom}(G, \mathbb{k})$ : for each $i$ and $d$ as above, the variety $\mathcal{R}_{d}^{i}(X, \mathbb{k})$ consists of those classes $z$ for which the dimension of $H^{i}(A, z)$ is at least $d$.

The tangent cone at 1 to $\mathcal{V}_{d}^{i}(X, \mathbb{C})$ is contained in $\mathcal{R}_{d}^{i}(X, \mathbb{C})$ [34], but, in general, the inclusion is strict $[37,22]$. We say that the exponential formula holds for $X$ (in degree $i \leq k$ and depth $d>0$ ) if the exponential map restricts to an isomorphism of analytic germs, $\exp :\left(\mathcal{R}_{d}^{i}(X, \mathbb{C}), 0\right) \stackrel{\simeq}{\longrightarrow}\left(\mathcal{V}_{d}^{i}(X, \mathbb{C}), 1\right)$. In this case, $\tau_{1}\left(\mathcal{V}_{d}^{i}(X, \mathbb{C})\right)=\mathcal{R}_{d}^{i}(X, \mathbb{C})$.

1.4. Characteristic varieties and $\Sigma$-invariants. For a variety $W \subseteq \operatorname{Hom}\left(G, \mathbb{C}^{\times}\right)$, let $\tau_{1}^{\mathbb{R}}(W) \subseteq \operatorname{Hom}(G, \mathbb{R})$ denote the real points on $\tau_{1}(W)$. For a subset $U \subseteq \operatorname{Hom}(G, \mathbb{R})$, let $U^{\complement}$ denote its complement. Under some mild assumptions, we relate in $\S 9$ the BNSR invariants and the homology jumping loci of a space $X$, as follows.

Theorem 1.1. Let $X$ be a connected $C W$-complex with finitely many cells up to dimension $k$, for some $k \geq 1$. Then, for each $q \leq k$, the following "exponential tangent cone upper bound" holds:

$$
\Sigma^{q}(X, \mathbb{Z}) \subseteq\left(\bigcup_{i \leq q} \tau_{1}^{\mathbb{R}}\left(\mathcal{V}_{1}^{i}(X, \mathbb{C})\right)\right)^{\complement}
$$


If, moreover, the exponential formula holds for $X$ up to degree $q$, and depth $d=1$, then the following "resonance upper bound" obtains:

$$
\Sigma^{q}(X, \mathbb{Z}) \subseteq\left(\bigcup_{i \leq q} \mathcal{R}_{1}^{i}(X, \mathbb{R})\right)^{\complement}
$$

When the group $G$ admits a classifying space $K(G, 1)$ satisfying the assumptions from Theorem 1.1, one may replace in (1) and (2) above $X$ by $G$ on the left-hand side, and $X$ by $K(G, 1)$ on the right-hand side.

This theorem answers a question raised by one of us at a Mini-Workshop held at Oberwolfach in August 2007, and recorded in the problems list of its proceedings [29].

Clearly, every additive character $\chi \in \operatorname{Hom}(G, \mathbb{R})$ factors as $\chi=\iota \circ \xi$, where $\xi: G \rightarrow \Gamma$ is a surjection onto a lattice in $\mathbb{R}$, and $\iota: \Gamma \hookrightarrow \mathbb{R}$ is the inclusion map. Since the NovikovSikorav completion is a rather complicated ring, we turn to a commutative approximation: the Novikov completion, $\widehat{\mathbb{Z}}_{\iota}$. This idea is certainly not new, as it has been used by Farber [27, Chapter 1] and Pajitnov [42] in related contexts. The main novelty consists in using our exponential tangent cone formalism, to obtain a computable, conceptually simpler, upper bound for the $\Sigma$-invariants, in terms of homology with twisted (rank 1) coefficients.

Our approach to the BNSR invariants via Novikov homology leads to further results, relating these invariants to the characteristic varieties over arbitrary fields. In $\S 10$, we prove the following theorem.

Theorem 1.2. Let $X$ be a connected $C W$-complex with finite $k$-skeleton ( $k \geq 1)$, and set $G=\pi_{1}(X)$.

(1) Let $\rho: G \rightarrow \mathbb{k}^{\times}$be a homomorphism such that $\rho \in \bigcup_{i \leq q} \mathcal{V}_{1}^{i}(X, \mathbb{k})$, for some $q \leq k$, and let $v: \mathbb{k}^{\times} \rightarrow \mathbb{R}$ be a valuation on $\mathbb{k}$ such that $v \circ \rho \neq 0$. Then $v \circ \rho \notin \Sigma^{q}(X, \mathbb{Z})$.

(2) Let $\chi=\iota \circ \xi: G \rightarrow \mathbb{R}$ be an additive character such that $-\chi \in \Sigma^{q}(X, \mathbb{Z})$, for some $q \leq k$, and let $\rho: \Gamma \rightarrow \mathbb{k}^{\times}$be a character which is not an algebraic integer. Then $\rho \circ \xi \notin \bigcup_{i \leq q} \mathcal{V}_{1}^{i}(X, \mathbb{k})$.

Part (1) of this theorem extends one of the main results from [18], proved by Delzant only for $\Sigma^{1}$, using a different approach.

1.5. Homology of free abelian covers. In $\S 6$, we refine the aforementioned result of Dwyer and Freed [25], as follows. Let $X$ be connected CW-complex with finite $k$-skeleton $(k \geq 1)$. Let us say $X$ satisfies condition $(*)_{k}$ if, for each $i \leq k$, the part of $\mathcal{V}_{1}^{i}(X, \mathbb{C})$ contained in the connected component of $1 \in \operatorname{Hom}\left(\pi_{1}(X), \mathbb{C}^{\times}\right)$is a union of subtori passing through 1 , and $T C_{1}\left(\mathcal{V}_{1}^{i}(X, \mathbb{C})\right)=\mathcal{R}_{1}^{i}(X, \mathbb{C})$.

Now let $\nu: \pi_{1}(X) \rightarrow \mathbb{Z}^{r}(r \geq 1)$ be an epimorphism, and $X^{\nu} \rightarrow X$ the corresponding Galois cover. If $r=1$, denote by $\nu_{\mathbb{C}} \in H^{1}(X, \mathbb{C})$ the corresponding cohomology class.

Theorem 1.3. With notation as above,

(1) If $r=1$, then $\bigoplus_{i \leq k} H_{i}\left(X^{\nu}, \mathbb{C}\right)$ is finite-dimensional if and only if $\nu_{\mathbb{C}}$ does not belong to any of the linear subspaces comprising $\bigcup_{i<k} \tau_{1}\left(\mathcal{V}_{1}^{i}(X, \mathbb{C})\right)$.

(2) If $r \geq 1$, and $X$ satisfies $(*)_{k}$, then $\bigoplus_{i \leq k} H_{i}\left(X^{\nu}, \overline{\mathbb{C}}\right)$ is finite-dimensional if and only if the pullback of $H^{1}\left(\mathbb{Z}^{r}, \mathbb{C}\right)$ along $\nu^{*}$ intersects $\bigcup_{i \leq k} \mathcal{R}_{1}^{i}(X, \mathbb{C})$ only at 0 . 
As a consequence, homological finiteness of regular, free abelian covers is an open condition in the appropriate Grassmanian, provided the above assumptions on the space $X$ are met. On the other hand, if $r>1$, and condition $(*)_{k}$ is violated, the finiteness property is no longer an open condition, as shown by an example from [25].

1.6. First applications. For the rest of the paper, we illustrate our results - in particular, bounds (1) and (2) from Theorem 1.1-with examples arising in a variety of geometric and topological contexts. The general inclusion (1) may well be strict, simply because there are situations when $\Sigma^{1} \neq-\Sigma^{1}$. Nevertheless, (1) can often be improved to better upper bounds.

We start by discussing such an improvement in $\S 11$. It is shown in [23] that the exponential formula holds for $X$, in degree $i=1$ and arbitrary depth $d$, whenever $G=\pi_{1}(X)$ is a 1-formal group, i.e., its Malcev Lie algebra is quadratic. In this case, (2) implies that $\Sigma^{1}(G) \subseteq \mathcal{R}_{1}^{1}(X, \mathbb{R})^{\complement}$, with equality when $X$ belongs to a large class of compact Kähler manifolds (see $\S 1.9$ below).

In $\S 12$, we consider the class of finitely generated nilpotent groups. If $G$ is such a group, we note that bound $(1)$ is sharp, that is, $\Sigma^{q}(G, \mathbb{Z})=\left(\bigcup_{i \leq q} \tau_{1}^{\mathbb{R}}\left(\mathcal{V}_{1}^{i}(K(G, 1), \mathbb{C})\right)\right)^{\complement}$, for all $q \geq 0$. On the other hand, bound (2) fails for many nilpotent groups, such as the Heisenberg group.

In $\S 13$, we analyze the behavior of (co)homology jumping loci and $\Sigma$-invariants with respect to product and coproduct operations. In particular, we discuss the product formula for BNSR invariants of groups. Originally conjectured by Bieri [6], this formula was recently proved by Bieri and Geoghegan [8]. We show in Theorem 13.6 that the class of groups of type $\mathrm{F}_{k}(k \geq 1)$ for which upper bound (1) holds as an equality, for all $q \leq k$, is closed under direct products.

1.7. Toric complexes. Given a simplicial complex $L$ on $n$ vertices, the toric complex $T_{L}$ is the subcomplex of the $n$-torus obtained by deleting the cells corresponding to the missing simplices of $L$. The fundamental group of $T_{L}$ is the right-angled Artin group $G_{\Gamma}$ attached to the graph $\Gamma$ defined as the 1-skeleton of $L$. A classifying space for $G_{\Gamma}$ is the toric complex $T_{\Delta_{\Gamma}}$, where $\Delta_{\Gamma}$ is the flag complex of $\Gamma$.

In $\S 14$, we find a perfect complementary match between the BNSR invariants and the cohomology jumping loci of a right-angled Artin group. The $\Sigma$-invariants of such groups (with coefficients in an arbitrary commutative ring), were computed by Meier, Meinert, and VanWyk in [39], with a more convenient description given later on by Bux and Gonzalez in [13]. The characteristic and resonance varieties of a toric complex $T_{L}$ were computed in [46], in terms of the algebraic combinatorics of the underlying simplicial complex $L$. In particular, the exponential formula holds for a toric complex, in arbitrary degree and depth. By Theorem 1.1(2),

$$
\Sigma^{k}\left(T_{L}, \mathbb{Z}\right) \subseteq\left(\bigcup_{i \leq k} \mathcal{R}_{1}^{i}\left(T_{L}, \mathbb{R}\right)\right)^{\complement}, \quad \text { for all } k \geq 0 .
$$

Using all this information, we show in Corollary 14.6 that

$$
\Sigma^{k}\left(G_{\Gamma}, \mathbb{R}\right)=\left(\bigcup_{i \leq k} \mathcal{R}_{1}^{i}\left(T_{\Delta_{\Gamma}}, \mathbb{R}\right)\right)^{\complement}
$$


for all graphs $\Gamma$, and for all $k \geq 0$. This equality recovers a result from [44], where we only dealt with the case $k=1$. (That special case, it should be said, provided much of the initial impulse for this work.) Finally, we describe in Corollary 14.7 natural torsionfreeness assumptions on the integral homology of certain subcomplexes of $\Delta_{\Gamma}$, which force upper bound (3) to become an equality, in the case when $L=\Delta_{\Gamma}$.

1.8. Artin kernels. In $\S 15$, we consider the regular, free abelian covers of a toric complex $T_{L}$. Given a homomorphism $\chi: G_{\Gamma} \rightarrow \mathbb{Z}^{r}$, the homological finiteness properties over an arbitrary field $\mathbb{k}$ of the corresponding cover, $T_{L}^{\chi}$, depend only on the position of a certain linear subspace determined by $\chi$, with respect to a subspace arrangement determined by $L$. This combinatorial dependence is made explicit in Theorem 15.2. In characteristic 0, things are even simpler: the vector space $\bigoplus_{i \leq q} H_{i}\left(T_{L}^{\chi}, \mathbb{Q}\right)$ is finite-dimensional if and only if the pullback of $H^{1}\left(\mathbb{Z}^{r}, \mathbb{Q}\right)$ along $\chi^{*}$ intersects $\bigcup_{i \leq q} \mathcal{R}_{1}^{i}\left(T_{L}, \mathbb{Q}\right)$ only at 0 .

Next, we focus on the Artin kernels $N_{\chi}=\operatorname{ker}\left(\chi: G_{\Gamma} \rightarrow \mathbb{Z}\right)$. Particularly interesting are the groups $N_{\Gamma}$ considered by Bestvina and Brady in [4], corresponding to the "diagonal" homomorphism $\nu$. For example, if $\Gamma$ is the 1-skeleton of the octahedron, then $N_{\Gamma}$ is none other than Stallings' group from [52]. In general, $N_{\Gamma}$ is finitely generated if and only if $\Gamma$ is connected, while $N_{\Gamma}$ is finitely presented if and only if $\Delta_{\Gamma}$ is simply-connected.

In previous work [46], we showed that the triviality of the monodromy action of $\mathbb{Z}$ on $\bigoplus_{i \leq k} H_{i}\left(N_{\chi}, \mathbb{Q}\right)$ can be tested in a purely combinatorial way. Moreover, if $H_{1}\left(N_{\chi}, \mathbb{Q}\right)$ is a trivial $\mathbb{Q} Z$-module, then $N_{\chi}$ is finitely generated. Here, we compute in Theorem 15.6 the jump loci $\mathcal{V}_{1}^{1}\left(N_{\chi}, \mathbb{C}\right)$ and $\mathcal{R}_{1}^{1}\left(N_{\chi}, \mathbb{C}\right)$, assuming trivial monodromy on $H_{1}\left(N_{\chi}, \mathbb{Q}\right)$, respectively, on $\bigoplus_{i \leq 2} H_{i}\left(N_{\chi}, \mathbb{Q}\right)$.

Keeping the trivial monodromy assumption on $H_{1}\left(N_{\chi}, \mathbb{Q}\right)$, we show in Theorem 15.8 that $\Sigma^{1}\left(N_{\chi}\right) \subseteq U^{\complement}$, where $U$ is the pullback to $N_{\chi}$ of a subspace arrangement in $H^{1}\left(G_{\Gamma}, \mathbb{R}\right)$, depending explicitly on the graph $\Gamma$. This enables us to construct in Example 15.11 finitely generated, yet not finitely presentable Artin kernels $N_{\chi}$ for which the BNS invariant $\Sigma^{1}\left(N_{\chi}\right)$ can be computed exactly.

1.9. Kähler and quasi-Kähler manifolds. We conclude in $\S 16$ with a discussion of the $\Sigma$-invariants of Kähler and quasi-Kähler manifolds, and of their fundamental groups.

The existence of a Kähler metric on a compact complex manifold $M$ puts strong constraints on its topology. For instance, $M$ must be a formal space [17], and thus, $G=\pi_{1}(M)$ must be 1 -formal. Hence, $\Sigma^{1}(G)$ lies in the complement of $\mathcal{R}_{1}^{1}(M, \mathbb{R})$, which, by [23], is a finite union of rationally defined linear subspaces. Now suppose $b_{1}(M)>0$ (otherwise, of course, $\Sigma^{1}(G)=\emptyset$ ). Using a recent result of Delzant [19], we show in Theorem 16.4 that $\Sigma^{1}(G)=\mathcal{R}_{1}^{1}(M, \mathbb{R})^{\complement}$ precisely when $M$ admits no elliptic pencils with multiple fibers.

Similar, yet weaker results hold when $X$ is a quasi-Kähler manifold (i.e., $X$ is the complement of a normal-crossing divisor in a compact Kähler manifold), and $G=\pi_{1}(X)$. The inclusion $\Sigma^{1}(G) \subseteq \mathcal{R}_{1}^{1}(X, \mathbb{R})^{\complement}$ fails in this generality. Nevertheless, we always have the upper bound

$$
\Sigma^{1}(G) \subseteq T C_{1}^{\mathbb{R}}\left(\mathcal{V}_{1}^{1}(X, \mathbb{C})\right)^{\complement},
$$

for a quasi-Kähler manifold $X$. We establish (5) by using a deep result of Arapura [2], which implies that all irreducible components of $\mathcal{V}_{1}^{1}(X, \mathbb{C})$ containing 1 are subtori. 
When $X$ is the complement of a complex hyperplane arrangement, another basic result, due to Esnault, Schechtman, and Viehweg [26], implies that the exponential formula holds for $X$, in arbitrary degree and depth. Consequently, we obtain the combinatorial upper bound

$$
\Sigma^{q}(X, \mathbb{Z}) \subseteq\left(\bigcup_{i \leq q} \mathcal{R}_{1}^{i}(X, \mathbb{R})\right)^{\complement}, \quad \text { for all } q \geq 0
$$

\section{Homological Finiteness PROPERTIES}

We start by recalling some standard finiteness notions, for both spaces and groups.

2.1. Finiteness length. Throughout, $X$ will be a connected CW-complex, with skeleta $\left\{X^{(k)}\right\}_{k \geq 0}$. Without loss of generality, we will assume $X$ has a single 0-cell, call it $x_{0}$. Furthermore, we will assume $X$ has finitely many 1-cells. Set

$$
\varkappa(X)=\sup \left\{k \mid X^{(k)} \text { is finite }\right\} .
$$

In the terminology of Bieri [6], this is the finiteness length of $X$. Note that $1 \leq \varkappa(X) \leq \infty$, with the upper bound attained precisely when $X$ is of finite-type.

2.2. Homology with twisted coefficients. Let $p: \widetilde{X} \rightarrow X$ be the universal cover of our CW-complex. The cell structure on $X$ lifts to a cell structure on $\widetilde{X}$. Fixing a lift $\tilde{x}_{0} \in p^{-1}\left(x_{0}\right)$ identifies the fundamental group $G=\pi_{1}\left(X, x_{0}\right)$ with the group of deck transformations of $\tilde{X}$, which permute the cells. Therefore, we may view the cellular cell complex $C_{\bullet}(\tilde{X}, \mathbb{Z})$, with differential $\tilde{\partial}$, as a chain complex of free left modules over the group ring $\mathbb{Z} G$.

Given a right $\mathbb{Z} G$-module $A$, consider the chain complex $C_{\bullet}(X, A):=A \otimes_{\mathbb{Z} G} C_{\bullet}(\widetilde{X}, \mathbb{Z})$, with differential id $A_{A} \otimes \tilde{\partial}$. The homology groups of $X$ with coefficients in $A$ are then defined as $H_{i}(X, A):=H_{i}\left(C_{\bullet}(X, A)\right)$.

For the purpose of computing homology with certain twisted coefficients, in the finiteness range of $X$, the following lemma allows us to replace $X$ by a finite $\mathrm{CW}$-complex.

Lemma 2.1. Given a $C W$-complex $X$ as above, and an integer $1 \leq k \leq \varkappa(X)$, there exists a finite $C W$-complex $Y$ of dimension at most $k+1$, with $Y^{(k)}=X^{(k)}$, and a map $f: Y \rightarrow X$ such that, for every commutative ring $S$, and every ring map $\mathbb{Z} G \rightarrow S$, the induced homomorphism, $f_{*}: H_{i}(Y, S) \rightarrow H_{i}(X, S)$, is an $S$-module isomorphism, for all $i \leq k$.

Proof. Let $X^{\text {ab }}$ be the maximal abelian cover of $X$, corresponding to the abelianization map ab: $G \rightarrow G_{\mathrm{ab}}$. The cellular chain complex $\left(C_{i}\left(X^{\mathrm{ab}}\right), \partial_{i}^{\mathrm{ab}}\right)_{i \geq 0}$ is a chain complex of free modules over the (commutative) Noetherian $\operatorname{ring} R=\mathbb{Z} G_{\mathrm{ab}}$. Since $C_{k}\left(X^{\mathrm{ab}}\right)$ is finitely generated as an $R$-module, the $R$-submodule $B_{k}\left(X^{\mathrm{ab}}\right)=\operatorname{im}\left(\partial_{k+1}^{\mathrm{ab}}\right)$ is also finitely generated, let's say, by the images of $(k+1)$-cells $e_{1}, \ldots, e_{r}$.

Set $Y:=X^{(k)} \cup e_{1} \cup \cdots \cup e_{r}$, a finite subcomplex of $X^{(k+1)}$. Let $f: Y \rightarrow X$ be the inclusion map. By construction, $f_{*}: H_{i}(Y, R) \rightarrow H_{i}(X, R)$ is an $R$-isomorphism, for all $i \leq k$. Since any ring map $\mathbb{Z} G \rightarrow S$ with $S$ commutative must factor through $R$, the conclusion follows. 
2.3. Finiteness properties of groups. A group $G$ is said to satisfy property $\mathrm{F}_{k}$, for some $k \geq 1$, if $G$ admits a classifying space $K(G, 1)$ with finite $k$-skeleton. If there is a finite-type (respectively, finite) $K(G, 1)$, then $G$ is of type $\mathrm{F}_{\infty}$ (respectively, $\mathrm{F}$ ). For example, finite groups are of type $\mathrm{F}_{\infty}$, while fundamental groups of closed, aspherical manifolds are of type $\mathrm{F}$. Note that the $\mathrm{F}_{1}$ property is equivalent to $G$ being finitely generated, while the $\mathrm{F}_{2}$ property is equivalent to $G$ being finitely presentable.

These notions have an algebraic analogue. Let $A$ be a module over a ring $R$. The $R$-module $A$ is said to be of type $\mathrm{FP}_{k}$ if there is a projective $R$-resolution $P_{\bullet} \rightarrow A$, with $P_{i}$ finitely generated for all $i \leq k$. Modules of type $\mathrm{FP}_{\infty}$ and $\mathrm{FP}$ are similarly defined.

Now let $G$ be a group, and $R$ a commutative ring with $0 \neq 1$. Then $G$ is said to satisfy property $\mathrm{FP}_{k}(R)$ if the trivial $R G$-module $R$ is of type $\mathrm{FP}_{k}$. The $\mathrm{FP}_{\infty}(R)$ and $\mathrm{FP}(R)$ properties are defined analogously. We will mostly deal with property $\mathrm{FP}_{k}=\mathrm{FP}_{k}(\mathbb{Z})$. If $G$ is of type $\mathrm{FP}_{k}$, then of course all homology groups $H_{i}(G, \mathbb{Z})$, for $i \leq k$, are finitely generated.

Clearly, if $G$ is of type $\mathrm{F}_{k}$, then $G$ is of type $\mathrm{FP}_{k}$. The converse also holds, provided $k=1$, or $G$ is finitely presentable, and $k<\infty$. However, as shown by Bestvina and Brady [4], $\mathrm{FP}_{k} \nRightarrow \mathrm{F}_{k}$, for any $k \geq 2$.

It is worth noting that the FP properties behave well with respect to group extensions.

Lemma 2.2 ([5], Proposition 2.7). Let $1 \rightarrow N \rightarrow G \rightarrow Q \rightarrow 1$ be an exact sequence of groups, and assume $N$ is of type $\mathrm{FP}_{\infty}$. Then $G$ is of type $\mathrm{FP}_{k}$ if and only if $Q$ is.

\section{Characteristic varieties and Alexander varieties}

In this section, we discuss two types of homology jumping loci associated to a space, and establish a tight relation between the two.

3.1. Characteristic varieties. As before, let $X$ be a connected CW-complex with finite $k$-skeleton, $k \geq 1$, and $G=\pi_{1}(X)$. Fix a field $\mathbb{k}$, and let $\mathbb{k}^{\times}$be its group of units. The group of $\mathbb{k}$-valued characters, $\operatorname{Hom}\left(G, \mathbb{k}^{\times}\right)$, parametrizes rank 1 local systems on $X$. Computing homology groups with coefficients in these local systems leads to a natural filtration of the character group by homology jumping loci.

Definition 3.1. The characteristic varieties of $X$ (over $\mathbb{k}$ ) are the Zariski closed sets

$$
\mathcal{V}_{d}^{i}(X, \mathbb{k})=\left\{\rho \in \operatorname{Hom}\left(G, \mathbb{k}^{\times}\right) \mid \operatorname{dim}_{\mathbb{k}} H_{i}\left(X, \mathbb{k}_{\rho}\right) \geq d\right\},
$$

defined for all integers $i$ and $d$ with $0 \leq i \leq \varkappa(X)$ and $d>0$.

Here $\mathbb{k}_{\rho}$ is the 1 -dimensional $\mathbb{k}$-vector space, viewed as a module over the group $\operatorname{ring} \mathbb{k} G$ via $g \cdot a=\rho(g) a$, for $g \in G$ and $a \in \mathbb{k}$. In each degree $i$, the varieties $\mathcal{V}_{d}^{i}=\mathcal{V}_{d}^{i}(X, \mathbb{k})$ define a descending filtration, $\mathcal{V}_{1}^{i} \supseteq \mathcal{V}_{2}^{i} \supseteq \cdots$, of the algebraic variety $\operatorname{Hom}\left(G, \mathbb{k}^{\times}\right)$. In degree 0 , it is easy to check that $\mathcal{V}_{1}^{0}(X, \mathbb{k})=\{1\}$, and $\mathcal{V}_{d}^{0}(X, \mathbb{k})=\emptyset$, for $d>1$.

In order to compute the characteristic varieties in degrees $i \leq k$, for some $k \leq \varkappa(X)$, we may assume that $X$ is a finite $\mathrm{CW}$-complex of dimension $k+1$. Indeed, in view of Lemma 2.1, there is a CW-complex $Y$ with these properties, and a map $f: Y \rightarrow X$ such that the induced morphism between character groups, $f^{*}: \operatorname{Hom}\left(\pi_{1}(X), \mathbb{k}^{\times}\right) \rightarrow \operatorname{Hom}\left(\pi_{1}(Y), \mathbb{k}^{\times}\right)$, is an isomorphism, identifying $\mathcal{V}_{d}^{i}(Y, \mathbb{k})$ with $\mathcal{V}_{d}^{i}(X, \mathbb{k})$, for all $i \leq k$ and all $d>0$. 
It is worth noting that the characteristic varieties only depend on the characteristic of the field of definition. More precisely, we have the following.

Lemma 3.2. Let $\mathbb{k} \subseteq \mathbb{K}$ be a field extension, and let $\operatorname{Hom}\left(G, \mathbb{k}^{\times}\right) \subseteq \operatorname{Hom}\left(G, \mathbb{K}^{\times}\right)$be the corresponding inclusion of character groups. Then

$$
\mathcal{V}_{d}^{i}(X, \mathbb{k})=\mathcal{V}_{d}^{i}(X, \mathbb{K}) \cap \operatorname{Hom}\left(G, \mathbb{k}^{\times}\right) .
$$

Proof. The characteristic varieties are determinantal varieties of matrices defined over $\mathbb{Z}$. The claim follows.

If $G$ is a group of type $\mathrm{F}_{k}(k \geq 1)$, we may define the characteristic varieties of $G$ as $\mathcal{V}_{d}^{i}(G, \mathbb{k}):=\mathcal{V}_{d}^{i}(K(G, 1), \mathbb{k})$, for $0 \leq i \leq k$ and $d>0$.

Lemma 3.3. Let $X$ be a connected $C W$-complex with finite $k$-skeleton $(k \geq 1)$, and $\widetilde{X}$ its universal cover. If $\pi_{i}(\tilde{X})=0$, for all $i \leq k$, then:

(i) $G=\pi_{1}(X)$ is of type $\mathrm{F}_{k}$.

(ii) $\mathcal{V}_{d}^{i}(X, \mathbb{k})=\mathcal{V}_{d}^{i}(G, \mathbb{k})$, for all $i \leq k$, all $d>0$, and all fields $\mathbb{k}$.

Proof. Using the assumption, we may construct a classifying space $K=K(G, 1)$ with $K^{(k+1)}=X^{(k+1)}$. The two claims follow at once.

In particular, for any connected CW-complex $X$ with finite 1-skeleton, the degree 1 characteristic varieties of $X$ depend only on its fundamental group:

$$
\mathcal{V}_{d}^{1}(X, \mathbb{k})=\mathcal{V}_{d}^{1}\left(\pi_{1}(X), \mathbb{k}\right), \quad \text { for all } d>0 .
$$

3.2. Alexander varieties. Let $X$ be a space as above, and let $\nu: G \rightarrow H$ be an epimorphism from $G=\pi_{1}\left(X, x_{0}\right)$ to an abelian group $H$ (which necessarily must be finitely generated). Denote by $X^{\nu} \rightarrow X$ the corresponding Galois cover of $X$, with group of deck transformations $H$.

Recall that every finitely generated module $A$ over the Noetherian ring $R=\mathbb{k} H$ has a finite presentation, say, $R^{m} \stackrel{M}{\longrightarrow} R^{n} \rightarrow A \rightarrow 0$. The $i$-th elementary ideal of $A$, denoted $E_{i}(A)$, is the ideal generated by the minors of size $n-i$ of the $n \times m$ matrix $M$, with the convention that $E_{i}(A)=R$ if $i \geq n$, and $E_{i}(A)=0$ if $n-i>m$.

For each $i \leq \varkappa(X)$, the homology group $H_{i}\left(X^{\nu}, \mathbb{k}\right)$ is a finitely generated $\mathbb{k} H$-module. The support loci of the elementary ideals of these modules define a filtration of the character group of $H$, which pulls back to $G$ via $\nu^{*}: \operatorname{Hom}\left(H, \mathbb{k}^{\times}\right) \rightarrow \operatorname{Hom}\left(G, \mathbb{k}^{\times}\right)$.

Definition 3.4. The Alexander varieties of $X$ (over $\mathbb{k}$ ) are the algebraic sets

$$
\mathcal{W}_{d}^{i}(X, \nu, \mathbb{k})=\nu^{*}\left(V\left(E_{d-1}\left(H_{i}\left(X^{\nu}, \mathbb{k}\right)\right)\right)\right),
$$

with $0 \leq i \leq \varkappa(X)$ and $d>0$.

In particular, $\mathcal{W}_{1}^{i}(X, \nu, \mathbb{k})=\nu^{*}\left(V\left(\operatorname{ann} H_{i}\left(X^{\nu}, \mathbb{k}\right)\right)\right)$ is the pull-back of the support of the $\mathbb{k} H$-module $H_{i}\left(X^{\nu}, \mathbb{k}\right)$. Given a group $G$ of type $\mathrm{F}_{k}$, one may define as before the Alexander varieties $\mathcal{W}_{d}^{i}(G, \nu, \mathbb{k})$ for $0 \leq i \leq k$, and prove the analogue of Lemma 3.3(ii).

Noteworthy is the case when $H=G_{\mathrm{ab}}$, and $\nu=\mathrm{ab}$; the corresponding modules, $H_{i}\left(X^{\mathrm{ab}}, \mathbb{k}\right)$, are the classical Alexander invariants of $X$. In particular, $H_{1}\left(X^{\mathrm{ab}}, \mathbb{k}\right)=$ 
$G^{\prime} / G^{\prime \prime} \otimes \mathbb{k}$. Another case of note is when $H$ is the maximal torsion-free abelian quotient of $G, G_{\mathrm{abf}}$, and $\nu=$ abf is the canonical projection. In this case, we simply write $\mathcal{W}_{d}^{i}(X, \mathbb{k}):=\mathcal{W}_{d}^{i}(X$, abf, $\mathbb{k})$.

Now suppose $G$ admits a finite presentation, say, $G=\left\langle x_{1}, \ldots, x_{n} \mid r_{1}, \ldots, r_{m}\right\rangle$. The associated Alexander matrix, $A_{G}=\left(\partial r_{i} / \partial x_{j}\right)^{\text {abf }}:\left(\mathbb{Z} G_{\text {abf }}\right)^{m} \rightarrow\left(\mathbb{Z} G_{\text {abf }}\right)^{n}$, is obtained by abelianizing the matrix of Fox derivatives of the relators, see [30]. The codimension $d$ minors of $A_{G}$ define the variety $\mathcal{W}_{d}^{1}(G, \mathbb{k})$, away from the trivial character 1.

Example 3.5. Consider the Laurent polynomial ring $\mathbb{Z}\left[\mathbb{Z}^{n}\right]=\mathbb{Z}\left[t_{1}^{ \pm 1}, \ldots, t_{n}^{ \pm 1}\right]$, and pick elements $v_{1}, \ldots, v_{n}$ satisfying the equation $\sum_{j=1}^{n}\left(t_{j}-1\right) v_{j}=0$. Let $F_{n}=\left\langle x_{1}, \ldots, x_{n}\right\rangle$ be the free group of rank $n$, with abelianization map ab: $F_{n} \rightarrow \mathbb{Z}^{n}, x_{j} \mapsto t_{j}$. By a result of R. Lyndon recorded in [30], there exists an element $r \in F_{n}^{\prime}$ such that $v_{j}=\left(\partial r / \partial x_{j}\right)^{\mathrm{ab}}$, for all $j$. For $n=2$, the commutator-relator group $G:=\left\langle x_{1}, x_{2} \mid r\right\rangle$ has first characteristic variety $\mathcal{V}_{1}^{1}(G, \mathbb{k})=\{1\} \cup V(\Delta) \subseteq\left(\mathbb{k}^{\times}\right)^{2}$, where $\Delta=\operatorname{gcd}\left(v_{1}, v_{2}\right)$ is the Alexander polynomial of $G$; see for instance [21].

3.3. Comparing the two sets of varieties. We adopt the spectral sequence approach of Dimca and Maxim from [20, 22.5$]$, in a broader context.

Recall $X$ is a connected CW-complex with $\varkappa(X) \geq 1$, and $G$ is its fundamental group. As before, let $\nu: G \rightarrow H$ be an epimorphism to an abelian group $H$. Consider the group ring $R=\mathbb{k} H$. A character $\rho: H \rightarrow \mathbb{k}^{\times}$corresponds to a ring map $\bar{\rho}: R \rightarrow \mathbb{k}$, which in turn corresponds to the maximal ideal $\mathfrak{m}=\operatorname{ker} \bar{\rho}$ in $R$. Denote by $M_{\mathfrak{m}}$ the localization of an $R$-module $M$ at $\mathfrak{m}$. Clearly, $\bar{\rho}$ factors through a ring morphism $\bar{\rho}_{\mathfrak{m}}: R_{\mathfrak{m}} \rightarrow \mathbb{k}$.

Let $\nu^{*}: \operatorname{Hom}\left(H, \mathbb{k}^{\times}\right) \rightarrow \operatorname{Hom}\left(G, \mathbb{k}^{\times}\right)$be the induced homomorphism. The key to comparing the characteristic and Alexander varieties is the Künneth spectral sequence associated to the chain complex $C_{*}\left(X, R_{\mathfrak{m}}\right)$ and the change-of-rings map $\bar{\rho}_{\mathfrak{m}}$ :

$$
E_{s, t}^{2}=\operatorname{Tor}_{s}^{R_{\mathfrak{m}}}\left(H_{t}\left(X^{\nu}, \mathbb{k}\right)_{\mathfrak{m}}, \mathbb{k}_{\bar{\rho}_{\mathfrak{m}}}\right) \Rightarrow H_{s+t}\left(X, \mathbb{k}_{\nu^{*} \rho}\right) .
$$

Theorem 3.6. For all $0 \leq k \leq \varkappa(X)$,

$$
\bigcup_{i=0}^{k} \mathcal{W}_{1}^{i}(X, \nu, \mathbb{k})=\operatorname{im}\left(\nu^{*}\right) \cap\left(\bigcup_{i=0}^{k} \mathcal{V}_{1}^{i}(X, \mathbb{k})\right) .
$$

Proof. The proof is by induction on $k$. Clearly, $H_{0}\left(X^{\nu}, \mathbb{k}\right)=\mathbb{k}$ is isomorphic to $R / I$, where $I$ is the augmentation ideal; thus, $\mathcal{W}_{1}^{0}(X, \nu, \mathbb{k})=\operatorname{supp} R / I=V(I)=\{1\}$. On the other hand, $\mathcal{V}_{1}^{0}(X, \mathbb{k})=\{1\}$, and so the two sides of (10) agree for $k=0$.

$(\supseteq)$ Suppose $\rho \notin \bigcup_{i=0}^{k} \operatorname{supp} H_{i}\left(X^{\nu}, \mathbb{k}\right)$. Then $E_{s, t}^{2}=0$, for $t \leq k$, and so $E_{s, t}^{\infty}=0$, for $t \leq k$. Consequently, $H_{i}\left(X, \mathbb{k}_{\nu^{*} \rho}\right)=0$, for all $i \leq k$, which means that $\rho \notin \bigcup_{i=0}^{k} \mathcal{V}_{1}^{i}(X, \mathbb{k})$.

$(\subseteq)$ Suppose $\rho \in \bigcup_{i=0}^{k} \operatorname{supp} H_{i}\left(X^{\nu}, \mathbb{k}\right)$. We may assume $\rho \notin \operatorname{supp} H_{t}\left(X^{\nu}, \mathbb{k}\right)$, for all $t<k$, and $\rho \in \operatorname{supp} H_{k}\left(X^{\nu}, \mathbb{k}\right)$, for otherwise we'd be done, by the induction hypothesis. These assumptions mean that $E_{s, t}^{2}=0$, for $t<k$, and $H_{k}\left(X^{\nu}, \mathbb{k}\right)_{\mathfrak{m}} \neq 0$. Thus:

$$
H_{k}\left(X, \mathbb{k}_{\nu^{*} \rho}\right)=E_{0, k}^{\infty}=E_{0, k}^{2}=H_{k}\left(X^{\nu}, \mathbb{k}\right)_{\mathfrak{m}} \otimes_{R_{\mathfrak{m}}} \mathbb{k}_{\bar{\rho}_{\mathfrak{m}}}=\frac{H_{k}\left(X^{\nu}, \mathbb{k}\right)_{\mathfrak{m}}}{\mathfrak{m} \cdot H_{k}\left(X^{\nu}, \mathbb{k}\right)_{\mathfrak{m}}}
$$

which is non-zero, by Nakayama's Lemma. Hence, $\nu^{*}(\rho) \in \mathcal{V}_{1}^{k}(X, \mathbb{k})$, and we're done. 
We will denote the character group $\operatorname{Hom}\left(G, \mathbb{k}^{\times}\right)$by $\mathbb{T}_{G}(\mathbb{k})$. The notation $\mathbb{T}_{G}^{0}(\mathbb{k})$ will be used for the subgroup abf* $\left(\mathbb{T}_{G_{\text {abf }}}(\mathbb{k})\right)$, where abf is the projection onto the maximal free abelian quotient of $G$. When $\mathbb{k}=\mathbb{C}$, we will simply write $\mathbb{T}_{G}$, respectively $\mathbb{T}_{G}^{0}$. The following is an immediate corollary of Theorem 3.6.

Corollary 3.7. For all $0 \leq k \leq \varkappa(X)$,

$$
\bigcup_{i=0}^{k} \mathcal{W}_{1}^{i}(X, \mathrm{ab}, \mathbb{k})=\bigcup_{i=0}^{k} \mathcal{V}_{1}^{i}(X, \mathbb{k}) \text {, and } \bigcup_{i=0}^{k} \mathcal{W}_{1}^{i}(X, \mathbb{k})=\mathbb{T}_{G}^{0}(\mathbb{k}) \cap\left(\bigcup_{i=0}^{k} \mathcal{V}_{1}^{i}(X, \mathbb{k})\right) \text {. }
$$

\section{TANGEnt CONES AND EXPONENTIAL TANGENT CONES}

In this section, we discuss two types of tangent cones associated to a Zariski closed subset of the character variety $\operatorname{Hom}\left(G, \mathbb{C}^{\times}\right)$: the usual kind, defined by the initial ideal, and a less standard one, coming from the exponential map. We start with the latter.

4.1. The exponential map. Let $G$ be a finitely generated group. Its character group, $\mathbb{T}_{G}=\operatorname{Hom}\left(G, \mathbb{C}^{\times}\right)$, may be identified with the cohomology group $H^{1}\left(G, \mathbb{C}^{\times}\right)$. Note that $\mathbb{T}_{G}$ is a complex algebraic group, with identity $1 \in \mathbb{T}_{G}$ the constant character $G \rightarrow \mathbb{C}^{\times}$, $g \mapsto 1$, and $\mathbb{T}_{G}^{0}$ is the connected component of $1 \in \mathbb{T}_{G}$.

Consider the complex vector space $\operatorname{Hom}(G, \mathbb{C})=H^{1}(G, \mathbb{C})$. Since $G$ is finitely generated, the dimension of this vector space, $n:=b_{1}(G)$, is finite. The exponential map $\mathbb{C} \rightarrow \mathbb{C}^{\times}, z \mapsto e^{z}$ is a group homomorphism. As such, it defines a coefficient homomorphism,

$$
\exp : H^{1}(G, \mathbb{C}) \rightarrow H^{1}\left(G, \mathbb{C}^{\times}\right)
$$

whose image is $\mathbb{T}_{G}^{0}$. Clearly, $\mathbb{T}_{G}^{0}=\left(\mathbb{C}^{\times}\right)^{n}$, an algebraic torus of dimension $n$.

4.2. Exponential tangent cones. We now recall a notion introduced in [23].

Definition 4.1. Let $W$ be a Zariski closed subset of $\mathbb{T}_{G}$. The exponential tangent cone of $W$ at 1 is the set

$$
\tau_{1}(W)=\left\{z \in H^{1}(G, \mathbb{C}) \mid \exp (t z) \in W, \text { for all } t \in \mathbb{C}\right\} .
$$

This construction enjoys several pleasant properties, some of which we record below.

Lemma 4.2. With notation as above,

(i) $\tau_{1}(W)$ is a homogeneous subvariety of $H^{1}(G, \mathbb{C})$.

(ii) $\tau_{1}(W)$ depends only on the analytic germ of $W$ at the identity.

(iii) $1 \notin W$ if and only if $\tau_{1}(W)=\emptyset$.

(iv) If $W_{1} \subseteq W_{2}$, then $\tau_{1}\left(W_{1}\right) \subseteq \tau_{1}\left(W_{2}\right)$.

(v) $\tau_{1}$ commutes with finite unions.

(vi) $\tau_{1}$ commutes with arbitrary intersections.

The proof is an exercise in the definitions. A deeper property of the exponential tangent cone is given in the following lemma.

Lemma 4.3 ([23]). For any subvariety $W \subseteq \mathbb{T}_{G}$, the exponential tangent cone $\tau_{1}(W)$ is a finite union of rationally defined linear subspaces of $H^{1}(G, \mathbb{C})$. 
Let us describe these subspaces concretely. Since $\tau_{1}$ commutes with intersections, it is enough to consider the case $W=V(f)$, where $f=\sum_{u \in S} c_{u} t_{1}^{u_{1}} \cdots t_{n}^{u_{n}}$ is a non-zero Laurent polynomial, with support $S \subseteq \mathbb{Z}^{n}$. We may assume $f(1)=0$, for otherwise, $\tau_{1}(W)=\emptyset$.

Let $\mathcal{P}$ be the set of partitions $p=S_{1} \amalg \cdots \amalg S_{r}$ of $S$, having the property that $\sum_{u \in S_{i}} c_{u}=0$, for $i=1, \ldots, r$. For each such partition, define

$$
L(p):=\left\{z \in \mathbb{C}^{n} \mid\langle u-v, z\rangle=0, \forall u, v \in S_{i}, \forall 1 \leq i \leq r\right\} .
$$

Clearly, $L(p)$ is a rational linear subspace in $\mathbb{C}^{n}$. Then, as shown in [23, Lemma 4.3],

$$
\tau_{1}(W)=\bigcup_{p \in \mathcal{P}} L(p) .
$$

Note that only maximal partitions contribute to this union. Indeed, if $p$ is a refinement of $q$, then $L(p) \supseteq L(q)$.

4.3. Tangent cones. For a Zariski closed subset $W \subseteq \mathbb{T}_{G}$, let $T C_{1}(W)$ be the tangent cone at 1 . The classical definition, due to Whitney, goes as follows. Let $I$ be the ideal in $\mathbb{C}\left\{z_{1}, \ldots, z_{n}\right\}$ defining the germ of $W$ at 1 , and let in $(I)$ be the ideal spanned by the initial forms of non-zero elements of $I$. Then $T C_{1}(W)$ is the affine cone in $H^{1}(G, \mathbb{C})=\mathbb{C}^{n}$ given by the zero-set of in $(I)$.

It is readily checked that $T C_{1}$ satisfies properties analogous to those satisfied by $\tau_{1}$, as enumerated in Lemma 4.2(i)-(v). We now compare the two definitions.

Proposition 4.4. Let $W$ be a subvariety of $\mathbb{T}_{G}$. Then:

(i) $\tau_{1}(W) \subseteq T C_{1}(W)$.

(ii) If all irreducible components of $W$ containing 1 are subtori, then $\tau_{1}(W)=T C_{1}(W)$.

Proof. Let $n=b_{1}(G)$, and identify $H^{1}(G, \mathbb{C})=\mathbb{C}^{n}$.

Part (i). Let $g \in \mathbb{C}\left\{z_{1}, \ldots, z_{n}\right\}$ be a non-zero analytic function vanishing on the germ of $W$ at 1 , where $z_{i}=t_{i}-1$. Let $g_{0}=i n(g)$ be its lowest homogeneous term. Suppose $z \in \tau_{1}(W)$. Then $g\left(e^{t z_{1}}-1, \ldots, e^{t z_{n}}-1\right)=0$, for all $t \in \mathbb{C}$ near 0 . Note that

$$
g\left(e^{t z_{1}}-1, \ldots, e^{t z_{n}}-1\right)=g_{0}\left(t z_{1}, \ldots, t z_{n}\right)+\text { higher order terms. }
$$

It follows that $g_{0}(z)=0$, and thus $z \in T C_{1}(W)$.

Part (ii). Using the fact that both $\tau_{1}$ and $T C_{1}$ commute with finite unions, we may assume $W$ is a single subtorus, passing through 1 . In this case, both $\tau_{1}(W)$ and $T C_{1}(W)$ are equal to the tangent space $T_{1}(W)$, and we are done.

The inclusion $\tau_{1}(W) \subseteq T C_{1}(W)$ can be strict even when 1 is a smooth point of $W$, as the next example shows.

Example 4.5. Consider the Laurent polynomial $f=t_{1}+t_{2}-2 \in \mathbb{Z}\left[t_{1}^{ \pm 1}, t_{2}^{ \pm 1}\right]$. Applying the method described in Example 3.5 to the polynomials $v_{1}=f\left(t_{2}-1\right)$ and $v_{2}=f\left(1-t_{1}\right)$, we may find a commutator-relator group $G=\left\langle x_{1}, x_{2} \mid r\right\rangle$ with Alexander polynomial $f$. We then have $\mathcal{V}_{1}^{1}(G, \mathbb{C})=\left\{\left(t_{1}, t_{2}\right) \in\left(\mathbb{C}^{\times}\right)^{2} \mid t_{1}+t_{2}=2\right\}$. Letting $W=\mathcal{V}_{1}^{1}(G, \mathbb{C})$, an easy calculation gives $\tau_{1}(W)=\left\{z \mid e^{t z_{1}}+e^{t z_{2}}=2\right\}=\{0\}$, whereas $T C_{1}(W)=\left\{z \mid z_{1}+z_{2}=0\right\}$. 


\section{Resonance varieties, formality, and the exponential Formula}

In this section, we discuss the jumping loci associated to the cohomology ring of a space. Under a formality assumption, these loci are related to the characteristic varieties via the exponential map.

5.1. Resonance varieties. Let $X$ be a connected CW-complex with finitely many $k$ cells, for $1 \leq k \leq \varkappa(X)$. Fix a field $\mathbb{k}$, and let $A=H^{*}(X, \mathbb{k})$ be the cohomology ring, with graded ranks the Betti numbers $b_{i}=\operatorname{dim}_{\mathbb{k}} A^{i}$. If char $\mathbb{k}=2$, assume additionally that $H_{1}(X, \mathbb{Z})$ has no 2 -torsion. (This mild condition presents no problems for us - it will be verified in all the examples we deal with here.) For each $a \in A^{1}$, we then have $a^{2}=0$. Thus, right-multiplication by $a$ defines a cochain complex

$$
(A, \cdot a): A^{0} \stackrel{a}{\longrightarrow} A^{1} \stackrel{a}{\longrightarrow} A^{2} \longrightarrow \cdots,
$$

known as the Aomoto complex. Let $\beta_{i}(A, a)=\operatorname{dim}_{\mathbb{k}} H^{i}(A, \cdot a)$ be the Betti numbers of this complex. The jumping loci for the Aomoto-Betti numbers define a natural filtration of the affine space $A^{1}=H^{1}(X, \mathbb{k})$.

Definition 5.1. The resonance varieties of $X$ (over $\mathbb{k}$ ) are the algebraic sets

$$
\mathcal{R}_{d}^{i}(X, \mathbb{k})=\left\{a \in A^{1} \mid \beta_{i}(A, a) \geq d\right\},
$$

with $0 \leq i \leq \varkappa(X)$ and $d>0$.

It is readily seen that each of these sets is a homogeneous algebraic subvariety of $A^{1}=$ $\mathbb{k}^{b_{1}}$. Indeed, $\beta_{i}(A, x a)=\beta_{i}(A, a)$, for all $x \in \mathbb{k}^{\times}$, and homogeneity follows. In each degree $i$ as above, the resonance varieties provide a descending filtration,

$$
H^{1}(X, \mathbb{k}) \supseteq \mathcal{R}_{1}^{i}(X, \mathbb{k}) \supseteq \cdots \supseteq \mathcal{R}_{b_{i}}^{i}(X, \mathbb{k}) \supseteq \mathcal{R}_{b_{i}+1}^{i}(X, \mathbb{k})=\emptyset .
$$

Note that, if $A^{i}=0$, then $\mathcal{R}_{d}^{i}(X, \mathbb{k})=\emptyset$, for all $d>0$. In degree 0 , it is straightforward to check that $\mathcal{R}_{1}^{0}(X, \mathbb{k})=\{0\}$, and $\mathcal{R}_{d}^{0}(X, \mathbb{k})=\emptyset$, for $d>1$.

The resonance varieties depend only on the characteristic of the ground field for the cohomology of $X$. More precisely, if $\mathbb{k} \subseteq \mathbb{K}$ is a field extension, then

$$
\mathcal{R}_{d}^{i}(X, \mathbb{k})=\mathcal{R}_{d}^{i}(X, \mathbb{K}) \cap H^{1}(X, \mathbb{k}) .
$$

If $G$ is a group of type $\mathrm{F}_{k}$ (and $G_{\mathrm{ab}}$ has no 2-torsion, if char $\mathbb{k}=2$ ), we may define the resonance varieties of $G$ as $\mathcal{R}_{d}^{i}(G, \mathbb{k}):=\mathcal{R}_{d}^{i}(K(G, 1), \mathbb{k})$, for $i \leq k$. It is readily checked that $\mathcal{R}_{d}^{1}(X, \mathbb{k})=\mathcal{R}_{d}^{1}\left(\pi_{1}(X), \mathbb{k}\right)$. The analogue of Lemma 3.3 holds, with the characteristic varieties replaced by resonance varieties.

Remark 5.2. Suppose $G$ is a finitely generated group (with torsion-free abelianization, if char $\mathbb{k}=2)$. Let $\nabla_{G}: \operatorname{Hom}_{\mathbb{k}}\left(H^{2}(G, \mathbb{k}), \mathbb{k}\right) \rightarrow H_{1}(G, \mathbb{k}) \wedge H_{1}(G, \mathbb{k})$ be the comultiplication map, dual to the cup-product map on $H^{1}(G, \mathbb{k})$. The holonomy Lie algebra of $G$, over the field $\mathbb{k}$, denoted $\mathfrak{h}(G, \mathbb{k})$, is the quotient of the free Lie algebra on the $\mathbb{k}$-vector space $H_{1}(G, \mathbb{k})$ by the ideal generated by the image of $\nabla_{G}$. Closely related is the infinitesimal Alexander invariant, $\mathfrak{b}(G, \mathbb{k}):=\mathfrak{h}^{\prime}(G, \mathbb{k}) / \mathfrak{h}^{\prime \prime}(G, \mathbb{k})$, viewed as a (finitely generated) module over the polynomial ring $S=\operatorname{Sym}\left(H_{1}(G, \mathbb{k})\right)$; see [43, Theorem 6.2] for more details. 
It turns out that the resonance variety $\mathcal{R}_{d}^{1}(G, \mathbb{k})$ coincides, away from the origin, with the zero set of the elementary ideal $E_{d-1}(\mathfrak{b}(G, \mathbb{k}))$. This is proved in [22, Lemma 4.2] when $G$ is finitely presented, but the argument works as well in this broader context.

5.2. The exponential formula. We now specialize to the case $\mathbb{k}=\mathbb{C}$. Let $G$ be the fundamental group of $X$. Consider the exponential map, exp: $\operatorname{Hom}(G, \mathbb{C}) \rightarrow \operatorname{Hom}\left(G, \mathbb{C}^{\times}\right)$, given by $\exp (\rho)(g)=e^{\rho(g)}$.

Definition 5.3. Let $k \leq \varkappa(X)$ be a positive integer. We say the exponential formula holds for $X$ (in degree $i \leq k$, and for depth $d>0$ ) if the exponential map restricts to an isomorphism of analytic germs,

$$
\exp :\left(\mathcal{R}_{d}^{i}(X, \mathbb{C}), 0\right) \stackrel{\simeq}{\longrightarrow}\left(\mathcal{V}_{d}^{i}(X, \mathbb{C}), 1\right) .
$$

Clearly, the exponential formula always holds in degree $i=0$, for arbitrary depth. The geometric motivation behind this definition comes from the following proposition.

Proposition 5.4. If the exponential formula (17) holds for $X$, then the following tangent cone formula also holds:

$$
T C_{1}\left(\mathcal{V}_{d}^{i}(X, \mathbb{C})\right)=\mathcal{R}_{d}^{i}(X, \mathbb{C}) .
$$

Moreover, $\tau_{1}\left(\mathcal{V}_{d}^{i}(X, \mathbb{C})\right)=T C_{1}\left(\mathcal{V}_{d}^{i}(X, \mathbb{C})\right)$, and so $\mathcal{R}_{d}^{i}(X, \mathbb{C})$ is a finite union of linear subspaces defined over $\mathbb{Q}$.

Proof. Let $W=\mathcal{V}_{d}^{i}(X, \mathbb{C})$. By Lemma $4.2, \tau_{1}(W)$ depends only on the analytic germ of $W$ at 1 . From the assumption, $\tau_{1}(W)=\mathcal{R}_{d}^{i}(X, \mathbb{C})$. But $\tau_{1}(W) \subseteq T C_{1}(W)$, by Proposition 4.4(i). The first two statements follow at once. The last statement now follows from Lemma 4.3.

Remark 5.5. For an arbitrary connected CW-complex with finite 1-skeleton,

$$
T C_{1}\left(\mathcal{V}_{d}^{i}(X, \mathbb{C})\right) \subseteq \mathcal{R}_{d}^{i}(X, \mathbb{C}),
$$

for all $i<\varkappa(X)$ and $d>0$. This follows from a result of Libgober [34] and Lemma 2.1. In general, though, the inclusion is strict; see [37] and [22].

5.3. Formality properties. Let $G$ be a finitely generated group. Following Sullivan [53], $G$ is said to be 1-formal if its Malcev completion (in the sense of Quillen [48]) is a quadratically presented, complete Lie algebra; we refer to [43] for more details. Examples of 1-formal groups are finitely generated Artin groups [33], and finitely presented BestvinaBrady groups [45]. If $b_{1}(G)=0$ or 1 , then $G$ is 1 -formal.

A finite-type CW-complex $X$ is said to be formal if its minimal model, as defined in [53], is quasi-isomorphic to $\left(H^{*}(X, \mathbb{Q}), 0\right)$. Examples of formal spaces are compact Kähler manifolds [17], rational cohomology tori, and complements of complex hyperplane arrangements. On the other hand, if $X$ is a nilmanifold, not homeomorphic to a torus, then $X$ is not formal. As noted by Sullivan, if $X$ is a formal space, then $\pi_{1}(X)$ is a 1-formal group.

The next result provides the main link between the 1-formality property of a group and its (co)homology jumping loci. 
Theorem 5.6 ([23]). Suppose $G$ is a 1-formal group. Then the exponential formula holds for $G$ in degree 1 , for all depths $d \geq 1$ :

$$
\exp :\left(\mathcal{R}_{d}^{1}(G, \mathbb{C}), 0\right) \stackrel{\simeq}{\longrightarrow}\left(\mathcal{V}_{d}^{1}(G, \mathbb{C}), 1\right) .
$$

In particular, $\tau_{1}\left(\mathcal{V}_{d}^{1}(G, \mathbb{C})\right)=T C_{1}\left(\mathcal{V}_{d}^{1}(G, \mathbb{C})\right)=\mathcal{R}_{d}^{1}(G, \mathbb{C})$, a union of linear subspaces defined over $\mathbb{Q}$.

\section{Homology of fRee ABelian COVERS}

In this section, we recall a beautiful result of Dwyer and Fried [25], which describes precisely how the Alexander varieties of a space control the homological finiteness properties of its free abelian covers. We then offer some refinements, and draw some consequences.

6.1. A criterion for homological finiteness. Let $X$ be a connected CW-complex (with single 0 -cell $\left.x_{0}\right)$, and $G=\pi_{1}\left(X, x_{0}\right)$. Fix a coefficient field $\mathbb{k}$, which we shall assume for the rest of this section to be algebraically closed, and write $H_{\leq k}(X, \mathbb{k}):=\bigoplus_{i=0}^{k} H_{i}(X, \mathbb{k})$.

Consider an epimorphism $\nu: G \rightarrow \mathbb{Z}^{r}$, and let $X^{\nu}$ be the corresponding $\mathbb{Z}^{r}$-cover. Denote by $\nu^{*}: \operatorname{Hom}\left(\mathbb{Z}^{r}, \mathbb{k}^{\times}\right) \rightarrow \operatorname{Hom}\left(G, \mathbb{k}^{\times}\right)$the induced homomorphism between character groups.

Theorem 6.1 (Dwyer and Fried [25]). Suppose $X$ has finitely many cells. Then, for all $k \geq 0$,

$$
\operatorname{dim}_{\mathbb{k}} H_{\leq k}\left(X^{\nu}, \mathbb{k}\right)<\infty \Longleftrightarrow \operatorname{im}\left(\nu^{*}\right) \cap\left(\bigcup_{i=0}^{k} \mathcal{W}_{1}^{i}(X, \mathbb{k})\right) \text { is finite. }
$$

Making use of the machinery developed in $\S 3$, we may relax the hypothesis of Theorem 6.1, from $X$ being finite to just $X^{(k)}$ being finite, and replace the Alexander varieties by the characteristic varieties. More precisely, we have the following corollary.

Corollary 6.2. Suppose $X$ has finitely many 1 -cells. Then, for all $0 \leq k \leq \varkappa(X)$,

$$
\operatorname{dim}_{\mathbb{k}} H_{\leq k}\left(X^{\nu}, \mathbb{k}\right)<\infty \Longleftrightarrow \operatorname{im}\left(\nu^{*}\right) \cap\left(\bigcup_{i=0}^{k} \mathcal{V}_{1}^{i}(X, \mathbb{k})\right) \text { is finite. }
$$

Proof. Corollary 3.7 of Theorem 3.6 yields $\bigcup_{i=0}^{k} \mathcal{W}_{1}^{i}(X, \mathbb{k})=\mathbb{T}_{G}^{0}(\mathbb{k}) \cap\left(\bigcup_{i=0}^{k} \mathcal{V}_{1}^{i}(X, \mathbb{k})\right)$. By Lemma 2.1, we may replace $X$ by a finite CW-complex with the same $k$-skeleton, without changing $H_{1}(X, \mathbb{Z})=G_{\mathrm{ab}}$, or the characteristic varieties in degrees $i \leq k$. The conclusion now follows from Theorem 6.1.

6.2. Homological finiteness and resonance. Under favorable circumstances, the homological finiteness properties of a free abelian cover can be tested by means of the resonance varieties. The next result proves Part (2) of Theorem 1.3 from the Introduction.

Proposition 6.3. Fix an integer $0 \leq k \leq \varkappa(X)$, and suppose that, for each $i \leq k$,

(a) $\mathcal{V}_{1}^{i}(X, \mathbb{C}) \cap \mathbb{T}_{G}^{0}$ is a union of subtori passing through 1 ;

(b) $T C_{1}\left(\mathcal{V}_{1}^{i}(X, \mathbb{C})\right)=\mathcal{R}_{1}^{i}(X, \mathbb{C})$. 
Let $\nu: G \rightarrow \mathbb{Z}^{r}$ be an epimorphism, and $\nu^{*}: H^{1}\left(\mathbb{Z}^{r}, \mathbb{C}\right) \rightarrow H^{1}(G, \mathbb{C})$ the induced homomorphism. Then $H_{\leq k}\left(X^{\nu}, \mathbb{C}\right)$ is finite dimensional if and only if

$$
\operatorname{im}\left(\nu^{*}\right) \cap\left(\bigcup_{i \leq k} \mathcal{R}_{1}^{i}(X, \mathbb{C})\right)=\{0\} .
$$

Proof. Write $\left(\bigcup_{i=0}^{k} \mathcal{V}_{1}^{i}(X, \mathbb{C})\right) \cap \mathbb{T}_{G}^{0}=\bigcup_{\alpha} T_{\alpha}$, with $T_{\alpha}$ subtori passing through 1 . Then:

$$
\begin{aligned}
& \operatorname{dim}_{\mathbb{C}} H_{\leq k}\left(X^{\nu}, \mathbb{C}\right)<\infty \underset{(\mathrm{i})}{\Longleftrightarrow} \nu^{*}\left(\operatorname{Hom}\left(\mathbb{Z}^{r}, \mathbb{C}^{\times}\right)\right) \cap\left(\bigcup_{\alpha} T_{\alpha}\right) \text { is finite } \\
& \underset{\text { (ii) }}{\Longleftrightarrow} \nu^{*}\left(H^{1}\left(\mathbb{Z}^{r}, \mathbb{C}\right)\right) \cap\left(\bigcup_{\alpha} T C_{1}\left(T_{\alpha}\right)\right)=\{0\} \\
& \Longleftrightarrow \nu^{*}\left(H^{1}\left(\mathbb{Z}^{r}, \mathbb{C}\right)\right) \cap\left(\bigcup_{i \leq k} T C_{1}\left(\mathcal{V}_{1}^{i}(X, \mathbb{C})\right)=\{0\}\right. \\
& \underset{\text { (iii) }}{\Longleftrightarrow} \nu^{*}\left(H^{1}\left(\mathbb{Z}^{r}, \mathbb{C}\right)\right) \cap\left(\bigcup_{i \leq k} \mathcal{R}_{1}^{i}(X, \mathbb{C})\right)=\{0\},
\end{aligned}
$$

where in (i) we used Corollary 6.2 , in (ii) we used $\operatorname{char}(\mathbb{C})=0$ and the hypothesis that $1 \in \bigcap_{\alpha} T_{\alpha}$, and in (iii) we used hypothesis (b).

The next example shows that it is really necessary to insist in hypothesis (a) that all subtori pass through the origin.

Example 6.4. Consider the group $G=\left\langle x_{1}, x_{2} \mid x_{1}^{2} x_{2} x_{1}^{-2} x_{2}^{-1}\right\rangle$, and let $X$ be the corresponding presentation 2-complex. Clearly, $H_{1}(X, \mathbb{Z})=\mathbb{Z}^{2}$, and the Alexander polynomial is $\Delta=t_{1}+1 \in \mathbb{Z}\left[t_{1}^{ \pm 1}, t_{2}^{ \pm 1}\right]$. Thus, $\mathcal{V}_{1}^{1}(X, \mathbb{C})$ consists of the origin 1 , together with the translated subtorus $\left\{\left(t_{1}, t_{2}\right) \in\left(\mathbb{C}^{\times}\right)^{2} \mid t_{1}=-1\right\}$.

Note that $\mathcal{R}_{1}^{1}(X, \mathbb{C})=\{0\}$, and so the tangent cone formula holds up to degree 1 , that is, $T C_{1}\left(\mathcal{V}_{1}^{i}(X, \mathbb{C})\right)=\mathcal{R}_{1}^{i}(X, \mathbb{C})$, for $i=0,1$. Now let $\nu=$ ab: $G \rightarrow \mathbb{Z}^{2}$. Then, $\operatorname{dim}_{\mathbb{C}} H_{1}\left(X^{\nu}, \mathbb{C}\right)=\infty$, by Corollary 6.2, although of course $\operatorname{im}\left(\nu^{*}\right) \cap \mathcal{R}_{1}^{1}(X, \mathbb{C})=\{0\}$.

6.3. Infinite cyclic covers. We now analyze in more detail the case $r=1$. As before, let $G=\pi_{1}(X)$. Given a homomorphism $\nu: G \rightarrow \mathbb{Z}$, the induced homomorphism in homology, $\nu_{*}: H_{1}(X, \mathbb{k}) \rightarrow H_{1}(\mathbb{Z}, \mathbb{k})=\mathbb{k}$, defines a cohomology class, $\nu_{\mathbb{k}} \in H^{1}(X, \mathbb{k})$. As noted in $[46, \S 4.4]$, we have $\nu_{\mathbb{k}}^{2}=0$, even if char $\mathbb{k}=2$.

The next result proves Part (1) of Theorem 1.3 from the Introduction.

Theorem 6.5. Let $\nu: G \rightarrow \mathbb{Z}$ be an epimorphism, and let $X^{\nu} \rightarrow X$ be the corresponding infinite cyclic cover. Then, for all $k \leq \varkappa(X)$,

$$
\operatorname{dim}_{\mathbb{C}} H_{\leq k}\left(X^{\nu}, \mathbb{C}\right)<\infty \Longleftrightarrow \nu_{\mathbb{C}} \notin \bigcup_{i=0}^{k} \tau_{1}\left(\mathcal{V}_{1}^{i}(X, \mathbb{C})\right) .
$$

Proof. Let $\nu^{*}: \mathbb{T}_{\mathbb{Z}} \rightarrow \mathbb{T}_{G}$ be the induced homomorphism on character tori. It is readily seen that the image of $\nu^{*}$ is the curve $C=\left\{\exp \left(t \nu_{\mathbb{C}}\right) \mid t \in \mathbb{C}\right\} \subseteq \mathbb{T}_{G}$.

By Corollary $6.2, H_{\leq k}\left(X^{\nu}, \mathbb{C}\right)$ is finite-dimensional if and only if $C$ intersects the variety $W=: \bigcup_{i=0}^{k} \mathcal{V}_{1}^{i}(X, \mathbb{C})$ in finitely many points. Since $C$ is irreducible, this happens precisely when $C \nsubseteq W$, which, by definition, is equivalent to $\nu_{\mathbb{C}} \notin \tau_{1}(W)$. Applying Lemma 4.2(v) ends the proof. 
Corollary 6.6. Suppose the exponential formula (17) holds for $X$, up to degree $k$, and for depth $d=1$. Then,

$$
\operatorname{dim}_{\mathbb{C}} H_{\leq k}\left(X^{\nu}, \mathbb{C}\right)<\infty \Longleftrightarrow \nu_{\mathbb{C}} \notin \bigcup_{i=0}^{k} \mathcal{R}_{1}^{i}(X, \mathbb{C}) .
$$

Proof. Combine Theorem 6.5 and Proposition 5.4.

Using Theorem 5.6 and Corollary 6.6, we obtain the following immediate consequence.

Corollary 6.7. Let $\nu: G \rightarrow \mathbb{Z}$ be an epimorphism. If $G$ is 1-formal, then

$$
b_{1}(\operatorname{ker} \nu)<\infty \Longleftrightarrow \nu_{\mathbb{C}} \notin \mathcal{R}_{1}^{1}(G, \mathbb{C}) \text {. }
$$

6.4. Homological finiteness as an open condition. Some of the above results can be interpreted qualitatively, as follows. Let $X$ be a CW-complex as before, and let $G=$ $\pi_{1}(X)$. We may view an epimorphism $\nu: G \rightarrow \mathbb{Z}^{r}$ as an element of $\operatorname{Gr}_{r}\left(H^{1}(X, \mathbb{Q})\right)$, the Grassmanian of $r$ planes in the vector space $H^{1}(X, \mathbb{Q})$.

Let $X^{\nu} \rightarrow X$ be the cover corresponding to $\nu$. As noted by Dwyer and Freed [25], if $X$ is a finite $\mathrm{CW}$-complex, the homological finiteness condition $\operatorname{dim}_{\mathbb{C}} H_{*}\left(X^{\nu}, \mathbb{C}\right)<\infty$ is an open condition for $r=1$. In the next Proposition, we recover their result, in a slightly more general and more precise form.

Proposition 6.8. Let $X$ be a connected $C W$-complex with finite 1-skeleton. Then, for every $k \leq \varkappa(X)$, the subset of $\operatorname{Gr}_{1}\left(H^{1}(X, \mathbb{Q})\right)$ defined by the condition $\operatorname{dim}_{\mathbb{C}} H_{\leq k}\left(X^{\nu}, \mathbb{C}\right)<$ $\infty$ is the complement of a finite union of projective subspaces.

Proof. By Lemma 4.3, the set $H^{1}(X, \mathbb{Q}) \cap \tau_{1}\left(\bigcup_{i=0}^{k} \mathcal{V}_{1}^{i}(X, \mathbb{C})\right)$ is a finite union of rational linear subspaces. In particular, its projectivized complement is a Zariski open subset in $\mathrm{Gr}_{1}\left(H^{1}(X, \mathbb{Q})\right)$. The claim now follows from Theorem 6.5.

Remark 6.9. For $r>1$, the above homological finiteness condition is no longer an open condition, as an example from [25] shows. In particular, one cannot strengthen Corollary 6.2 to the analogue of Theorem 6.5 , in the case when $r>1$.

\section{Bieri-Neumann-Strebel-Renz invariants}

In this section, we review the definition of the $\Sigma$-invariants of a group $G$, following BieriNeumann-Strebel [10] and Bieri-Renz [11], and discuss some of their basic properties.

7.1. $\Sigma$-invariants. Let $G$ be a finitely generated group. Pick a finite generating set for $G$, and let $\mathcal{C}(G)$ be the corresponding Cayley graph. Given an additive real character $\chi: G \rightarrow \mathbb{R}$, let $\mathcal{C}_{\chi}(G)$ be the full subgraph on vertex set $G_{\chi}=\{g \in G \mid \chi(g) \geq 0\}$. In [10], Bieri, Neumann, and Strebel defined a subset $\Sigma^{1}(G)$ of $\operatorname{Hom}(G, \mathbb{R}) \backslash\{0\}$, nowadays called the BNS-invariant of $G$, as follows.

Definition 7.1 ([10]). The set $\Sigma^{1}(G)$ consists of those non-zero homomorphisms $\chi: G \rightarrow$ $\mathbb{R}$ for which the graph $\mathcal{C}_{\chi}(G)$ is connected. 
Clearly, $\Sigma^{1}(G)$ is a conical subset of the vector space $\operatorname{Hom}(G, \mathbb{R})=H^{1}(G, \mathbb{R})$. It turns out that the BNS invariant $\Sigma^{1}(G)$ is an open subset of $\operatorname{Hom}(G, \mathbb{R})$, and that this subset does not depend on the choice of generating set for $G$, see [10].

In [11], Bieri and Renz generalized the BNS invariant, by defining a sequence of "homological" invariants, $\left\{\Sigma^{r}(G, R)\right\}_{r \geq 0}$, for any commutative ring $R$ with $1 \neq 0$ (viewed as a trivial $R G$-module).

Definition 7.2 ([11]). The set $\Sigma^{r}(G, R)$ consists of those nontrivial homomorphisms $\chi \in \operatorname{Hom}(G, \mathbb{R})$ for which $R$ is of type $\mathrm{FP}_{r}$, when viewed as a module over the monoid $\operatorname{ring} R G_{\chi}$.

The BNSR invariants $\Sigma^{r}(G, R)$ form a descending chain of open subsets of $\Sigma^{0}(G, R)=$ $\operatorname{Hom}(G, \mathbb{R}) \backslash\{0\}$. Clearly, if $b_{1}(G)=0$, then $\operatorname{Hom}(G, \mathbb{R})=\{0\}$, and so $\Sigma^{r}(G, R)=\emptyset$, for all $r \geq 0$.

As noted in [11], if $\Sigma^{r}(G, \mathbb{Z}) \neq \emptyset$, then $G$ is of type $\mathrm{FP}_{r}$. We will be especially interested in the invariants $\Sigma^{r}(G, \mathbb{Z})$ and $\Sigma^{r}(G, \mathbb{k})$, with $\mathbb{k}$ a field. There is always an inclusion $\Sigma^{r}(G, \mathbb{Z}) \subseteq \Sigma^{r}(G, \mathbb{k})$, but this inclusion may be strict.

One may also define "homotopical" invariants, $\Sigma^{r}(G)$. If $G$ is of type $\mathrm{F}_{k}$, the two kinds of geometric invariants are related by Hurewicz-type formulas: $\Sigma^{1}(G)=\Sigma^{1}(G, R)$, for all $R$, and $\Sigma^{r}(G)=\Sigma^{2}(G) \cap \Sigma^{r}(G, \mathbb{Z})$, for $2 \leq r \leq k$.

7.2. Symmetry. If $M^{3}$ is a closed 3-manifold, and $G=\pi_{1}(M)$, then $\Sigma^{1}(G)=-\Sigma^{1}(G)$, as proved in $[10$, Corollary F]. In general, though, the $\Sigma$-invariants are not symmetric about the origin, as the next, well-known example shows.

Example 7.3. Consider the Baumslag-Solitar group $G=\left\langle x_{1}, x_{2} \mid x_{1} x_{2} x_{1}^{-1}=x_{2}^{2}\right\rangle$. Clearly, $G_{\mathrm{ab}}=\mathbb{Z}$ is freely generated by $x_{1}$. Upon this identification, Theorem 7.3 from [11] implies that $\Sigma^{1}(G)=\mathbb{R}_{<0}$. In particular, $\Sigma^{1}(G) \neq-\Sigma^{1}(G)$.

For future reference, let us note the following straightforward criterion for symmetry of the BNSR invariants. Suppose there is an automorphism $\alpha: G \rightarrow G$ such that $\alpha_{*}: H_{1}(G, \mathbb{R}) \rightarrow H_{1}(G, \mathbb{R})$ equals - id. Then $\Sigma^{r}(G, R)=-\Sigma^{r}(G, R)$, for all $r \geq 1$.

7.3. Finiteness properties of kernels. Much of the importance of the $\Sigma$-invariants rests with the fact that they control the finiteness properties of kernels of projections to abelian quotients. Given a normal subgroup $N \triangleleft G$, write $S(G, N)=\{\chi \in \operatorname{Hom}(G, \mathbb{R}) \backslash\{0\} \mid$ $\chi(N)=0\}$.

Theorem 7.4 ([10], [11]). Let $G$ be a finitely generated group, and let $N \triangleleft G$ be a normal subgroup with $G / N$ abelian. Then

(i) $N$ is of type $\mathrm{F}_{k}$ if and only if $S(G, N) \subseteq \Sigma^{k}(G)$.

(ii) $N$ is of type $\mathrm{FP}_{k}$ if and only if $S(G, N) \subseteq \Sigma^{k}(G, \mathbb{Z})$.

In particular, if $\chi: G \rightarrow \mathbb{Z}$ is an epimorphism, then $N=\operatorname{ker}(\chi)$ is finitely generated if and only if $\{ \pm \chi\} \subseteq \Sigma^{1}(G)$. In view of the theorem of Stallings [51] mentioned in $\S 1.2$, this implies the following: A closed 3-manifold $M$ fibers over $S^{1}$ if and only if $\Sigma^{1}\left(\pi_{1}(M)\right) \neq \emptyset$. 


\section{NovikOV HOMOLOGY}

In this section, we recall how the $\Sigma$-invariants of a group $G$ may be reinterpreted in terms of the vanishing of certain Tor groups. In turn, this interpretation opens the way to extending the definition of these invariants to spaces.

We start by reviewing a notion introduced by S. P. Novikov in the early 1980s, and later generalized by J.-Cl. Sikorav [50]. We refer to M. Farber's book [27] for a comprehensive treatment, and to R. Bieri [7] for further details.

8.1. The Novikov-Sikorav completion. Let $G$ be a finitely generated group, and let $\chi: G \rightarrow \mathbb{R}$ be an additive character. The Novikov-Sikorav completion of the group ring $\mathbb{Z} G$ with respect to $\chi$, denoted $\widehat{\mathbb{Z}}_{\chi}$, consists of all formal sums $\lambda=\sum_{i} n_{i} g_{i}$, with $n_{i} \in \mathbb{Z}$ and $g_{i} \in G$, having the property that the set of indices $i$ for which $n_{i} \neq 0$ and $\chi\left(g_{i}\right) \geq c$ is finite, for each $c \in \mathbb{R}$. With componentwise addition, and multiplication defined by $\left(\sum_{i} n_{i} g_{i}\right) \cdot\left(\sum_{j} m_{j} h_{j}\right)=\sum_{i, j}\left(n_{i} m_{j}\right) g_{i} h_{j}$, the Novikov-Sikorav completion becomes a ring. Clearly, $\widehat{\mathbb{Z}}_{\chi}$ contains $\mathbb{Z} G$ as a subring, and thus acquires a natural $G$-module structure. For more details, see [27, p. 52].

The above construction is a ring completion, in the following sense. For each integer $s$, let $F_{s}$ be the abelian subgroup of $\mathbb{Z} G$ generated by those $g \in G$ with $\chi(g) \geq s$. Requiring the decreasing filtration $\left\{F_{s}\right\}_{s \in \mathbb{Z}}$ to form a basis of open neighborhoods of 0 defines a topology on $\mathbb{Z} G$, compatible with the ring structure. It turns out that

$$
\widehat{\mathbb{Z} G}_{-\chi}={\underset{\leftarrow}{\mathrm{lim}}}_{\mathrm{Z}} \mathbb{Z} G / F_{s},
$$

the completion of $\mathbb{Z} G$ with respect to this topology. Moreover, the inclusion map between the respective topological rings, $\kappa_{\chi}: \mathbb{Z} G \hookrightarrow \widehat{\mathbb{Z} G}_{-\chi}$, is the structural map for this completion.

The Novikov-Sikorav completion enjoys the following functoriality property. Let $\phi: G \rightarrow$ $H$ be a homomorphism, and $\bar{\phi}: \mathbb{Z}^{G} \rightarrow \mathbb{Z}^{H}$ its linear extension to formal sums. If $\chi: H \rightarrow \mathbb{R}$ is a character, then $\bar{\phi}$ restricts to a (topological) ring morphism, $\hat{\phi}: \widehat{\mathbb{Z} G}_{\chi \circ \phi} \rightarrow \widehat{\mathbb{Z} H}_{\chi}$, between the corresponding completions.

8.2. Generalized $\Sigma$-invariants. In his thesis [50], J.-Cl. Sikorav reinterpreted the $\Sigma$ invariants of a finitely generated group $G$ in terms of the vanishing of its homology groups, with coefficients in $\widehat{\mathbb{Z}}_{\chi}$. Let us record here this basic result, in a form due to Bieri (Theorem 2 from [7], proved in an appendix by P. Schweitzer).

Theorem 8.1 ([7]). Let $G$ be a group of type $\mathrm{FP}_{k}(k \geq 1)$. For a character $\chi \in$ $\operatorname{Hom}(G, \mathbb{R}) \backslash\{0\}$, and an integer $q \leq k$,

$$
\chi \in \Sigma^{q}(G, \mathbb{Z}) \Longleftrightarrow \operatorname{Tor}_{i}^{\mathbb{Z} G}\left(\widehat{\mathbb{Z} G}_{-\chi}, \mathbb{Z}\right)=0, \text { for all } i \leq q .
$$

This result naturally leads to the following definition.

Definition 8.2. Let $X$ be a connected CW-complex with finite 1-skeleton, and fundamental group $G=\pi_{1}(X)$. For each $q \geq 0$, set

$$
\Sigma^{q}(X, \mathbb{Z}):=\left\{\chi \in \operatorname{Hom}(G, \mathbb{R}) \backslash\{0\} \mid H_{\leq q}\left(X, \widehat{\mathbb{Z} G}_{-\chi}\right)=0\right\} .
$$


Clearly, if $G$ is a group of type $\mathrm{FP}_{k}$, then $\Sigma^{q}(G, \mathbb{Z})=\Sigma^{q}(K(G, 1), \mathbb{Z})$, for all $q \leq k$. Thus, this definition generalizes the usual definition of the BNSR invariants. At the same time, Proposition 7 from [28] implies that the above definition coincides with the one introduced by Farber, Geoghegan, and Schütz, whenever $X$ is a finite CW-complex.

For the record, let us note that the analogue of Lemma 3.3 holds for the $\Sigma$-invariants of spaces and groups, in the form given below, and with essentially the same proof.

Lemma 8.3. Let $X$ be a connected $C W$-complex with finite $k$-skeleton ( $k \geq 1$ ), with universal cover $\tilde{X}$ and fundamental group $G$. If $\pi_{i}(\tilde{X})=0$, for all $i \leq k$, then $\Sigma^{i}(X, \mathbb{Z})=$ $\Sigma^{i}(G, \mathbb{Z})$, for all $i \leq k$.

8.3. Rational Novikov ring. As before, let $G$ be a finitely generated group, and $\chi: G \rightarrow$ $\mathbb{R}$ a non-zero homomorphism. Let $\Gamma$ be the image of $\chi$. The group $\Gamma$ is a lattice, say, $\Gamma \cong \mathbb{Z}^{r}$, with $r>0$. Moreover, $\chi$ factors as $\chi=\iota \circ \xi$, where $\xi: G \rightarrow \Gamma$ is an epimorphism, and $\iota: \Gamma \hookrightarrow \mathbb{R}$ is a monomorphism. By functoriality of Novikov-Sikorav completion, we have the commuting square on the left side of the diagram,

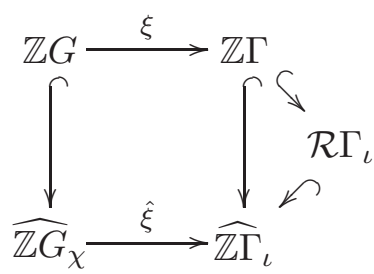

The ring $\mathbb{Z} \Gamma$ is a Laurent polynomial ring, while its completion, $\widehat{\mathbb{Z}}_{\iota}$, is a principal ideal domain (PID). There is a very useful intermediary ring, $\mathcal{R} \Gamma_{\iota}$, which Farber [27] calls the rational Novikov ring of $\Gamma$ (with respect to $\iota$ ). The construction of $\mathcal{R} \Gamma_{\iota}$ goes as follows.

A Laurent polynomial $p=\sum_{\gamma} n_{\gamma} \gamma \in \mathbb{Z} \Gamma$ is called $\iota$-monic if the greatest element in $\iota(\operatorname{supp}(p))$ is 0 , and $n_{0}=1$. By [27, Lemma 1.9], every such polynomial is invertible in $\widehat{\mathbb{Z}}_{\iota}$. The ring $\mathcal{R} \Gamma_{\iota}$ is then the localization of $\mathbb{Z} \Gamma$ at the multiplicative subset $S$ of all $\iota$-monic polynomials. As shown in [27, Lemma 1.15], the ring $\mathcal{R} \Gamma_{\iota}=S^{-1} \mathbb{Z} \Gamma$ is a PID. In fact, as shown by Pajitnov in [41, Theorem 1.4], $\mathcal{R} \Gamma_{\iota}$ is a Euclidean ring.

8.4. $\Sigma$-invariants and Novikov Betti numbers. Now let $X$ be a connected CWcomplex, with finite $k$-skeleton $(k \geq 1)$. Let $G=\pi_{1}(X)$, and consider a character $\chi: G \stackrel{\xi}{\rightarrow} \Gamma \stackrel{\iota}{\rightarrow} \mathbb{R}$ factored as above. Taking advantage of the fact that the ring $\mathcal{R} \Gamma_{\iota}$ is both a $G$-module and a PID, one may define the Novikov Betti numbers of $X$ as

$$
b_{i}(X, \chi):=\operatorname{rank}_{\mathcal{R} \Gamma_{\iota}} H_{i}\left(X, \mathcal{R} \Gamma_{\iota}\right), \quad \text { for } i \leq k .
$$

Remark 8.4. Using Lemma 2.1, we may assume in this definition that $X$ is a finite CW-complex, which is the assumption that Farber makes in [27].

Proposition 8.5. Let $X$ be a $C W$-complex with finite 1-skeleton. Then, for all $q \leq \varkappa(X)$,

$$
-\chi \in \Sigma^{q}(X, \mathbb{Z}) \Longrightarrow H_{i}\left(X, \mathcal{R} \Gamma_{\iota}\right)=0, \text { for all } i \leq q .
$$

In particular, $b_{i}(X, \chi)=0$, for $i \leq q$. 
Proof. Set $G=\pi_{1}(X)$. Let $-\chi \in \Sigma^{q}(X, \mathbb{Z})$, and fix $i \leq q$. By Definition 8.2, the group $H_{i}\left(X, \widehat{\mathbb{Z}}_{\chi}\right)$ vanishes. Using the change-of-rings spectral sequence associated to the ring $\operatorname{morphism} \hat{\xi}: \widehat{\mathbb{Z}}_{\chi} \rightarrow \widehat{\mathbb{Z}}_{\iota}$,

$$
E_{s t}^{2}=\operatorname{Tor}_{s}^{\widehat{\mathbb{Z} G}}\left(\widehat{\mathbb{Z}}_{\iota}, H_{t}\left(X, \widehat{\mathbb{Z} G}_{\chi}\right)\right) \Rightarrow H_{s+t}\left(X, \widehat{\mathbb{Z}}_{\iota}\right),
$$

we find that $H_{i}\left(X, \widehat{\mathbb{Z}}_{\iota}\right)$ also vanishes. Using [27, Proposition 1.29], we conclude that $H_{i}\left(X, \mathcal{R} \Gamma_{\iota}\right)=0$.

\section{9. $\Sigma$-INVARIANTS AND JUMPING LOCI OVER $\mathbb{C}$}

In this section, we prove our main Theorem, relating the BNSR invariants of a space (or of a group) to its homology jump loci. We start with a result asserting that nonmembership in the exponential tangent cones to the characteristic varieties is equivalent to vanishing of the respective Novikov-Betti numbers. This improves on a similar vanishing result, implied by Farber's Theorem 1.50 from [27].

9.1. Tangent cones and Novikov-Betti numbers. Let $X$ be a connected $\mathrm{CW}$-complex with finite $k$-skeleton $(k \geq 1)$, and $G=\pi_{1}(X)$ its fundamental group. Identify $\operatorname{Hom}(G, \mathbb{R})$ and $H^{1}(G, \mathbb{R})$. For an algebraic subset $W \subseteq \mathbb{T}_{G}$, denote by $\tau_{1}^{\mathbb{R}}(W)=\tau_{1}(W) \cap H^{1}(G, \mathbb{R})$ the set of real points on the exponential tangent cone of $W$ (and similarly for $T C_{1}$ ).

Theorem 9.1. Let $\chi: G \rightarrow \mathbb{R}$ be a non-zero character. Then, for each $0 \leq q \leq k$,

$$
\chi \notin \tau_{1}^{\mathbb{R}}\left(\bigcup_{i \leq q} \mathcal{V}_{1}^{i}(X, \mathbb{C})\right) \Longleftrightarrow b_{i}(X, \chi)=0, \text { for all } i \leq q
$$

Proof. Factor $\chi=\iota \circ \xi$, with $\xi: G \rightarrow \Gamma$ and $\iota: \Gamma \hookrightarrow \mathbb{R}$ as before. Let $\mathcal{O}_{\mathbb{C}}$ be the ring of holomorphic functions on $\mathbb{C}$. Define a group homomorphism $\phi: \Gamma \rightarrow \mathcal{O}_{\mathbb{C}}^{\times}$, with values in the units of $\mathcal{O}_{\mathbb{C}}$, by $\phi(\gamma)(t)=\exp (t \iota(\gamma))$, and extend $\phi$ linearly to a ring morphism $\bar{\phi}: \mathbb{C} \Gamma \rightarrow \mathcal{O}_{\mathbb{C}}$. The functions $\exp \left(t \iota\left(\gamma_{1}\right)\right), \ldots, \exp \left(t \iota\left(\gamma_{m}\right)\right)$ are linearly independent over $\mathbb{C}$, for distinct $\gamma_{1}, \ldots, \gamma_{m}$; hence, the map $\bar{\phi}$ is injective. For a fixed $t \in \mathbb{C}$, let ev ${ }_{t}: \mathcal{O}_{\mathbb{C}} \rightarrow \mathbb{C}$ be the evaluation map.

Since $R=\mathcal{R} \Gamma_{\iota}$ is a PID, we may decompose the finitely generated $R$-module $H_{i}(X, R)$ as $R^{b_{i}} \oplus \operatorname{Tors}_{i}$, where $b_{i}=b_{i}(X, \chi)$. Write $\bigoplus_{i=0}^{q} \operatorname{Tors}_{i}=\bigoplus_{\alpha} R / f_{\alpha} R$, for some finite collection of non-zero Laurent polynomials $f_{\alpha} \in \mathbb{Z} \Gamma$.

$(\Leftarrow)$ Suppose $b_{i}=0$, for all $i \leq q$. Define

$$
D_{0}:=\bigcup_{\alpha} V\left(\bar{\phi}\left(f_{\alpha}\right)\right) \cup \bigcup_{p: p \text { is } \iota \text {-monic }} V(\bar{\phi}(p)) .
$$

Since $\bar{\phi}$ is injective, $D_{0}$ is a countable subset of $\mathbb{C}$. Pick $t \in \mathbb{C} \backslash D_{0}$. Since $t \notin \bigcup_{p} V(\bar{\phi}(p))$, there is a ring map $\beta: R \rightarrow \mathbb{C}$ making the following diagram commute.

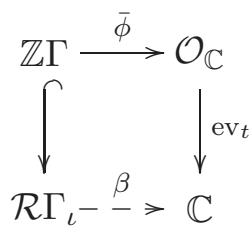


Consider the local coefficient system $\mathbb{C}_{\rho}$ on $X$, given by the character $\rho: G \rightarrow \mathbb{C}^{\times}$, $\rho(g)=\exp (t \chi(g))$. Fix an integer $i \leq q$. By the Universal Coefficient Theorem and diagram (25),

$$
H_{i}\left(X, \mathbb{C}_{\rho}\right)=\operatorname{Tor}_{0}^{R}\left(H_{i}(X, R), \mathbb{C}_{\beta}\right) \oplus \operatorname{Tor}_{1}^{R}\left(H_{i-1}(X, R), \mathbb{C}_{\beta}\right),
$$

where $\mathbb{C}_{\beta}$ denotes $\mathbb{C}$ viewed as an $\mathcal{R} \Gamma_{\iota}$-module, via $\beta$.

Since $t \notin \bigcup_{\alpha} V\left(\bar{\phi}\left(f_{\alpha}\right)\right)$, both summands in (26) vanish. Hence, $\rho \notin \mathcal{V}_{1}^{i}(X, \mathbb{C})$, and so $\chi \notin \tau_{1}^{\mathbb{R}}\left(\mathcal{V}_{1}^{i}(X, \mathbb{C})\right)$.

$(\Rightarrow)$ For the converse, define

$$
D_{1}:=D_{0} \cup \psi^{-1}\left(\bigcup_{i \leq q} \mathcal{V}_{1}^{i}(X, \mathbb{C})\right),
$$

where $\psi: \mathbb{C} \rightarrow \mathbb{T}_{G}$ is given by $\psi(t)=\rho$, with $\rho(g)=\exp (t \chi(g))$ as before. Notice that

$$
\begin{aligned}
\chi \notin \tau_{1}^{\mathbb{R}}\left(\bigcup_{i \leq q} \mathcal{V}_{1}^{i}(X, \mathbb{C})\right) & \Longleftrightarrow\{\exp (t \chi) \mid t \in \mathbb{C}\} \not \subset \bigcup_{i \leq q} \mathcal{V}_{1}^{i}(X, \mathbb{C}) \\
& \Longleftrightarrow \psi^{-1}\left(\bigcup_{i \leq q} \mathcal{V}_{1}^{i}(X, \mathbb{C})\right) \neq \mathbb{C} .
\end{aligned}
$$

The set $\psi^{-1}\left(\bigcup_{i<q} \mathcal{V}_{1}^{i}(X, \mathbb{C})\right)$ is the zero locus of a finite set of global holomorphic functions on $\mathbb{C}$; thus, it must be countable. Hence, $D_{1}$ is countable.

Now pick $t \in \mathbb{C} \backslash D_{1}$, and fix an integer $i \leq q$. Since $t \notin \bigcup_{p} V(\bar{\phi}(p))$, we may decompose $H_{i}\left(X, \mathbb{C}_{\rho}\right)$ as in $(26)$. Since $\rho=\psi(t) \notin \mathcal{V}_{1}^{i}(X, \mathbb{C})$, the homology group $H_{i}\left(X, \mathbb{C}_{\rho}\right)$ must vanish. Since $H_{i}(X, R)=R^{b_{i}} \oplus \operatorname{Tors}_{i}$, the right side of (26) contains $\mathbb{C}^{b_{i}}$ as a direct summand. Hence, $b_{i}=0$, and we are done.

9.2. Upper bounds for the $\Sigma$-invariants. We are now ready to state and prove our main results, partly summarized in the Introduction as Theorem 1.1. Each of these results gives an upper bound on the BNSR invariants of a space, or of a group, starting in all generality, and then under certain hypotheses. As these hypotheses get more specific, the upper bound gets smaller.

Theorem 9.2. Fix an integer $k \geq 1$.

(1) Let $X$ be a connected $C W$-complex with finite $k$-skeleton. Then, for every $q \leq k$,

$$
\Sigma^{q}(X, \mathbb{Z}) \subseteq\left(\tau_{1}^{\mathbb{R}}\left(\bigcup_{i \leq q} \mathcal{V}_{1}^{i}(X, \mathbb{C})\right)\right)^{\complement} .
$$

(2) Let $G$ be a group of type $\mathrm{F}_{k}$. Then, for every $q \leq k$,

$$
\Sigma^{q}(G, \mathbb{Z}) \subseteq\left(\tau_{1}^{\mathbb{R}}\left(\bigcup_{i \leq q} \mathcal{V}_{1}^{i}(G, \mathbb{C})\right)\right)^{\complement}
$$

Proof. Follows from Proposition 8.5, Theorem 9.1, Lemma 4.2(i), and the remarks made after Definition 8.2. 
Corollary 9.3. With notation as in Theorem 9.2, suppose that, for each $i \leq q$, all the components of $\mathcal{V}_{1}^{i}(X, \mathbb{C})$ (respectively, $\mathcal{V}_{1}^{i}(G, \mathbb{C})$ ), passing through 1 are subtori. Then,

$$
\Sigma^{q}(X, \mathbb{Z}) \subseteq\left(\bigcup_{i \leq q} T C_{1}^{\mathbb{R}}\left(\mathcal{V}_{1}^{i}(X, \mathbb{C})\right)\right)^{\complement}, \text { resp. }, \quad \Sigma^{q}(G, \mathbb{Z}) \subseteq\left(\bigcup_{i \leq q} T C_{1}^{\mathbb{R}}\left(\mathcal{V}_{1}^{i}(G, \mathbb{C})\right)\right)^{\complement}
$$

Proof. Follows from Theorem 9.2, Lemma 4.2(v), and Proposition 4.4(ii).

Corollary 9.4. With notation as in Theorem 9.2, suppose the exponential formula (17) holds for $X$ (respectively, $G$ ), up to degree $q$, and for depth $d=1$. Then,

$$
\Sigma^{q}(X, \mathbb{Z}) \subseteq\left(\bigcup_{i \leq q} \mathcal{R}_{1}^{i}(X, \mathbb{R})\right)^{\complement}, \text { resp. }, \quad \Sigma^{q}(G, \mathbb{Z}) \subseteq\left(\bigcup_{i \leq q} \mathcal{R}_{1}^{i}(G, \mathbb{R})\right)^{\complement} .
$$

Proof. Follows from Theorem 9.2, Proposition 5.4, and formula (16).

Note that all upper bounds (28)-(31) are symmetric. In particular, they cannot be sharp when $\Sigma^{q}$ is not symmetric, as happens for the Baumslag-Solitar group from Example 7.3.

\section{0. $\Sigma$-INVARIANTS AND JUMPING LOCI IN ARBITRARY CHARACTERISTIC}

In this section, we relate the BNSR invariants of a space to its jump loci for homology with rank 1 coefficients in an arbitrary field $\mathbb{k}$. Throughout, $X$ will be a connected CWcomplex with finite $k$-skeleton $(k \geq 1)$, and $G=\pi_{1}(X)$ will be its fundamental group.

10.1. Valuations. We begin by constructing elements in the complement of $\Sigma^{q}(X, \mathbb{Z})$, starting from multiplicative, $\mathbb{k}$-valued characters belonging to the characteristic varieties of $X$. To achieve this, we use $\mathbb{R}$-valuations on $\mathbb{k}$, following an idea of Bieri and Groves [9].

The next result proves Part (1) of Theorem 1.2 from the Introduction.

Theorem 10.1. Let $\rho: G \rightarrow \mathbb{k}^{\times}$be a multiplicative character such that $\rho \in \bigcup_{i \leq q} \mathcal{V}_{1}^{i}(X, \mathbb{k})$, for some $q \leq k$. Let $v: \mathbb{k}^{\times} \rightarrow \mathbb{R}$ be the homomorphism defined by a valuation $v$ on $\mathbb{k}$, and set $\chi=v \circ \rho$. If the additive character $\chi: G \rightarrow \mathbb{R}$ is non-zero, then $\chi \notin \Sigma^{q}(X, \mathbb{Z})$.

Proof. Endow $\mathbb{Z} G$ with the topological ring structure defined by the character $\chi$, as explained in $\S 8.1$. Pick a real number $a \in(0,1)$, and put on $\mathbb{k}$ the topology associated to the absolute value $a^{v}$; in this way, $\mathbb{k}$ becomes a topological ring. Let $\widehat{\mathbb{k}}$ be the (topological) completion of $\mathbb{k}$. Then $\widehat{\mathbb{k}}$ is a field, and the structural morphism, $\kappa_{\mathbb{k}}: \mathbb{k} \hookrightarrow \widehat{\mathbb{k}}$, is a field extension.

Let $\bar{\rho}: \mathbb{Z} G \rightarrow \mathbb{k}$ be the ring morphism defined by $\rho$. It is easy to check that $\bar{\rho}$ is continuous with respect to the above topologies. Hence, $\bar{\rho}$ induces a morphism of topological rings, $\hat{\rho}$, between the respective completions, making the following diagram commute:

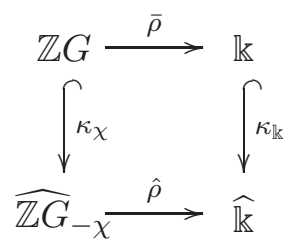

Now suppose $\chi \in \Sigma^{q}(X, \mathbb{Z})$. Changing coefficients from $\widehat{\mathbb{Z}}_{-\chi}$ to $\widehat{\mathbb{k}}$ via $\hat{\rho}$, we find that $H_{i}\left(X, \widehat{\mathbb{k}}_{\rho}\right)=0$, for all $i \leq q$. By Lemma 3.2, $\rho \notin \bigcup_{i \leq q} \mathcal{V}_{1}^{i}(X, \mathbb{k})$, a contradiction. 
Remark 10.2. For a finitely generated group $G$, Theorem 10.1 - when applied to the classifying space $X=K(G, 1)$, with $q=k=1$, and $v$ a discrete valuation-recovers Proposition 1 from [18], proved by Delzant using a different method, based on $G$-actions on trees.

10.2. Algebraic integers. Next, we examine the converse question: how to produce multiplicative $\mathbb{k}$-characters with trivial (twisted) homology, starting from elements in $\Sigma^{q}$. We will need the following lemma.

Lemma 10.3. Let $\Lambda$ be a commutative ring, $\mathbb{k}$ a field, and $\rho: \Lambda \rightarrow \mathbb{k}$ a ring morphism. If $\operatorname{Tor}_{s}^{\Lambda}(\mathbb{k}, N) \neq 0$, for some $\Lambda$-module $N$ and some $s \geq 0$, then $\rho(\Delta)=0$, for every $\Delta \in \operatorname{ann}_{\Lambda}(N)$.

Proof. Consider the homothety $\mu_{\Delta}$, acting $\Lambda$-linearly on $\Lambda$-modules. Since $\Delta$ annihilates $N$, this homothety acts as the zero map on $\operatorname{Tor}_{s}^{\Lambda}(\mathbb{k}, N)$.

Now pick a free $\Lambda$-resolution, $P_{\bullet} \rightarrow N$, where $P_{\bullet}=\Lambda \otimes_{\mathbb{Z}} C_{\bullet}$. Clearly, $\mu_{\Delta}$ acts on $\operatorname{Tor}_{s}^{\Lambda}(\mathbb{k}, N)=H_{s}\left(\mathbb{k} \otimes_{\mathbb{Z}} C_{\bullet}\right)$ as the $\mathbb{k}$-homothety $\mu_{\rho(\Delta)}$. Since $\operatorname{Tor}_{s}^{\Lambda}(\mathbb{k}, N) \neq 0$ and $\mathbb{k}$ is a field, we must have $\rho(\Delta)=0$.

As before, let $X$ be a connected CW-complex with finite $k$-skeleton $(k \geq 1)$, and $G=\pi_{1}(X)$. Given a non-trivial additive character $\chi \in \operatorname{Hom}(G, \mathbb{R})$, factor it as $\chi=\iota \circ \xi$, where $\xi: G \rightarrow \Gamma$ is the co-restriction of $\chi$ onto $\Gamma:=\operatorname{im}(\chi)$, and $\iota: \Gamma \hookrightarrow \mathbb{R}$ is the inclusion.

A multiplicative character $\rho: \Gamma \rightarrow \mathbb{k}^{\times}$is called an algebraic integer if $\Delta(\rho)=0$, for some Laurent polynomial $\Delta=\sum_{\gamma} n_{\gamma} \gamma \in \mathbb{Z} \Gamma$ with the property that $n_{\gamma_{0}}=1$, where $\gamma_{0}$ is the greatest element of $\iota(\operatorname{supp}(\Delta))$. Compare with §8.3, and [27, Definition 1.53].

The next result - inspired in part by Theorem 1.54 from [27] - completes the proof of Theorem 1.2 from the Introduction.

Theorem 10.4. Let $\chi=\iota \circ \xi: G \rightarrow \mathbb{R}$ be an additive character such that $-\chi \in \Sigma^{q}(X, \mathbb{Z})$, for some $q \leq k$. Let $\rho: \Gamma \rightarrow \mathbb{k}^{\times}$be a multiplicative character which is not an algebraic integer. Then $\xi^{*} \rho \notin \bigcup_{i \leq q} \mathcal{V}_{1}^{i}(X, \mathbb{k})$.

Proof. By Proposition 8.5, $H_{i}\left(X, \mathcal{R} \Gamma_{\iota}\right)=0$, for all $i \leq q$. In view of Lemma 2.1, we may assume, without loss of generality, that $X$ is a finite $\mathrm{CW}$-complex, since the ring $\mathcal{R} \Gamma_{\iota}$ is commutative. By [27, Proposition 1.34(2)], there is an element $\Delta=\sum_{\gamma} n_{\gamma} \gamma$ in the ring $\Lambda:=\mathbb{Z} \Gamma$, so that $\Delta$ has top coefficient $n_{\gamma_{0}}=1$, and $\Delta$ annihilates $H_{i}(X, \Lambda)$, for all $i \leq q$.

Consider now the spectral sequence associated to the change of rings map $\bar{\rho}: \Lambda \rightarrow \mathbb{k}$,

$$
E_{s t}^{2}=\operatorname{Tor}_{s}^{\Lambda}\left(\mathbb{k}_{\rho}, H_{t}(X, \Lambda)\right) \Rightarrow H_{s+t}\left(X, \mathbb{k}_{\xi^{*} \rho}\right) .
$$

Since, by assumption, the character $\rho$ is not an algebraic integer, Lemma 10.3 implies that $E_{s t}^{2}=0$, for all $t \leq q$ and $s \geq 0$. Consequently, $H_{i}\left(X, \mathbb{k}_{\xi^{*} \rho}\right)=0$, for all $i \leq q$, showing that $\xi^{*} \rho \notin \bigcup_{i \leq q} \mathcal{V}_{1}^{i}(X, \mathbb{k})$.

\section{1. $\Sigma$-INVARIANTS, 1-FORMALITY, AND DEFICIENCY}

For the remainder of this paper, we will give various examples for which the bounds derived in Section 9 hold, some with equality. We start in this section with a particularly manageable case - that of 1-formal groups - and discuss the relationship between $\Sigma$-invariants and deficiency. 
11.1. $\Sigma$-invariants of 1 -formal groups. Recall from Theorem 5.6 that the exponential formula holds in degree 1 , whenever $G$ is a 1 -formal group. It follows from Proposition 5.4 that $\mathcal{R}_{d}^{1}(G, \mathbb{C})$ is the union of a rationally defined subspace arrangement. Corollary 9.4 then yields the following.

Proposition 11.1. Suppose $G$ is a 1-formal group. Then the BNS invariant of $G$ is contained in the complement of a finite union of linear subspaces defined over $\mathbb{Q}$ :

$$
\Sigma^{1}(G) \subseteq \mathcal{R}_{1}^{1}(G, \mathbb{R})^{\complement} .
$$

A nice example where the above inclusion holds as an equality is provided by the McCool groups of basis-conjugating automorphisms, see D. Cohen [14].

In general, though, inclusion (34) is strict. For instance, take $G$ to be the BaumslagSolitar group from Example 7.3. Then $G$ is 1 -formal, since $b_{1}(G)=1$; yet $\Sigma^{1}(G) \neq$ $\mathcal{R}_{1}^{1}(G, \mathbb{R})^{\complement}$, since $\Sigma^{1}(G)$ is not symmetric about the origin.

In favorable situations, Proposition 11.1 allows us to determine exactly the BNS invariant of a group.

Corollary 11.2. Suppose $G$ is a 1 -formal group, and $\mathcal{R}_{1}^{1}(G, \mathbb{R})=H^{1}(G, \mathbb{R})$. Then $\Sigma^{1}(G)=\emptyset$.

This approach yields a quick application to 3-manifolds.

Corollary 11.3. Let $M$ be a closed, orientable 3-manifold, and let $G=\pi_{1}(M)$. Suppose $b_{1}(M)$ is even, and $G$ is 1-formal. Then $\Sigma^{1}(G)=\emptyset$ (and thus, $M$ does not fiber over $S^{1}$ ).

Proof. Since $b_{1}(M)$ is even, we have $\mathcal{R}_{1}^{1}(G, \mathbb{C})=H^{1}(G, \mathbb{C})$, by [24, Proposition 4.2]. The conclusion follows from Corollary 11.2.

If $b_{1}(M)$ is odd, the 3-manifold $M$ may of course fiber over the circle, even if $G$ is 1formal, but there are restrictions on what the algebraic monodromy can be. These aspects are pursued in [47], in a more general setting.

11.2. $\Sigma$-invariants and deficiency. Suppose $G$ is a finitely presented group. Its deficiency, denoted $\operatorname{def}(G)$, is the maximum value of the difference between the number of generators and the number of relations, taken over all finite presentations of $G$.

It turns out that there is a strong connection between the $\Sigma$-invariants of a group and its deficiency. For instance, Bieri, Neumann, and Strebel proved in [10, Theorem 7.2] the following: if $\operatorname{def}(G) \geq 2$, then $\Sigma^{1}(G)=\emptyset$. In [12], K. Brown gave an explicit algorithm for computing the BNS invariant of a 1-relator group $G$. In [11], Bieri and Renz reinterpreted this algorithm in terms of Fox calculus, and showed that, for 1-relator groups, $\Sigma^{r}(G, \mathbb{Z})=\Sigma^{1}(G)$, for all $r \geq 2$. For more on this subject, see Bieri [7].

Now let $X$ be a connected CW-complex with finite 2-skeleton, and denote by $c_{i}$ the number of $i$-cells of $X$, for $i=1,2$. The proposition below provides a simple class of examples where our general upper bound is optimal. For groups with deficiency at least 2, this proposition recovers the result of Bieri-Neumann-Strebel mentioned above.

Proposition 11.4. If $c_{1}-c_{2}>1$, then $\Sigma^{1}(X, \mathbb{Z})=\left(\tau_{1}^{\mathbb{R}}\left(\mathcal{V}_{1}^{1}(X, \mathbb{C})\right)\right)^{\complement}=\emptyset$. 
Proof. Let $\rho \in \operatorname{Hom}\left(\pi_{1}(X), \mathbb{C}^{\times}\right)$be a character. The chain complex computing the homology of $X$ with coefficients in $\mathbb{C}_{\rho}$ ends in $\mathbb{C}^{c_{2}} \rightarrow \mathbb{C}^{c_{1}} \rightarrow \mathbb{C}$. Therefore, $\operatorname{dim}_{\mathbb{C}} H_{1}\left(X, \mathbb{C}_{\rho}\right)$ is bounded below by $c_{1}-c_{2}-1>0$, and so $\rho \in \mathcal{V}_{1}^{1}(X, \mathbb{C})$. The conclusion follows from Theorem 9.2.

\section{Finitely Generated Nilpotent GROUPS}

In this section, we delineate another large class of groups for which the "exponential tangent cone upper bound" for the $\Sigma$-invariants is, in fact, an equality.

12.1. Finiteness properties. A group $G$ is said to be nilpotent if its lower central series, defined inductively by $\gamma_{1} G=G$ and $\gamma_{i+1} G=\left[\gamma_{i} G, G\right]$ for $i \geq 1$, where [, ] stands for the group commutator, terminates in finitely many steps. Clearly, subgroups and factor groups of nilpotent groups are again nilpotent.

Lemma 12.1. Let $G$ be a finitely generated nilpotent group. Then $G$ is of type $\mathrm{FP}_{\infty}$.

Proof. Let $\operatorname{Tors}(G)$ be the torsion subgroup of $G$. From the assumptions, it follows that $\operatorname{Tors}(G)$ is finite, hence of type $\mathrm{F}_{\infty}$. The quotient group, $Q=G / \operatorname{Tors}(G)$, is a torsionfree, finitely generated nilpotent group. As such, $Q$ admits as classifying space a compact nilmanifold of the form $M=\mathbb{R}^{n} / Q$; in particular, $Q$ is of type F. By Lemma 2.2, $G$ is of type $\mathrm{FP}_{\infty}$.

12.2. Jumping loci. The characteristic and resonance varieties of finitely generated nilpotent groups were studied in [36]. The former admit a very simple description.

Theorem $12.2([36])$. Let $G$ be a finitely generated nilpotent group. Then

$$
\mathcal{V}_{d}^{i}(G, \mathbb{C})= \begin{cases}\{1\} & \text { if } b_{i}(G) \geq d, \\ \emptyset & \text { otherwise. }\end{cases}
$$

On the other hand, the resonance varieties of such groups can be just about as complicated as possible. For example, as noted in [36, Remark 2.4], given any finitely generated group $H$, there is a 2 -step nilpotent group $G$ such that $\mathcal{R}_{d}^{1}(G, \mathbb{C})=\mathcal{R}_{d}^{1}(H, \mathbb{C})$, for all $d \geq 1$ : simply take $G=H / \gamma_{3} H$.

12.3. $\Sigma$-invariants. For completeness, we include a proof for the following result, which is presumably folklore.

Proposition 12.3. Let $G$ be a finitely generated nilpotent group. Then

$$
\Sigma^{q}(G, \mathbb{Z})=H^{1}(G, \mathbb{R}) \backslash\{0\}, \quad \text { for all } q \geq 0 .
$$

Proof. Any subgroup $H<G$ is again a finitely generated nilpotent group. By Lemma 12.1, $H$ is of type $\mathrm{FP}_{\infty}$. The claim follows at once from Theorem 7.4(ii).

Comparing the two answers from Theorem 12.2 and Proposition 12.3, we see that, in this case, the inclusion guaranteed by Theorem 9.2 is actually an equality.

Corollary 12.4. Let $G$ be a finitely generated nilpotent group. Then

$$
\Sigma^{q}(G, \mathbb{Z})=\left(\tau_{1}^{\mathbb{R}}\left(\bigcup_{i \leq q} \mathcal{V}_{1}^{i}(G, \mathbb{C})\right)\right)^{\complement}, \quad \text { for all } q \geq 0
$$


On the other hand, the "resonance upper bound" from Proposition 11.1 does not hold for all groups in this class, as the next example shows.

Example 12.5. Let $G=F_{2} / \gamma_{3} F_{2}$ be the Heisenberg group. It is readily seen that the cupproduct map on $H^{1}(G, \mathbb{Z})=\mathbb{Z}^{2}$ is zero, and so $\mathcal{R}_{1}^{1}(G, \mathbb{R})=\mathbb{R}^{2}$. In view of Proposition 12.3 , the inclusion $\Sigma^{1}(G) \subseteq \mathcal{R}_{1}^{1}(G, \mathbb{R})^{\complement}$ completely fails in this case. This observation recovers the well-known fact that $G$ is not a 1-formal group.

\section{Products And COproducts}

In this section, we analyze how the various invariants we studied so far behave under the product and coproduct operations for spaces and groups, and the extent to which our bounds for the $\Sigma$-invariants carry over under those operations.

13.1. Jumping loci of products. Let $X_{1}$ and $X_{2}$ be two CW-complexes, each one with a single 0-cell (which we will take as the basepoint), and finitely many 1-cells. Denote by $G_{1}$ and $G_{2}$ the corresponding fundamental groups. Fix a field $\mathbb{k}$; if char $\mathbb{k}=2$, assume $H_{1}\left(X_{j}, \mathbb{Z}\right)$ is torsion free, to have the resonance varieties of $X_{1}$ and $X_{2}$ defined. The jumping loci of the product CW-complex $X_{1} \times X_{2}$ can be computed in terms of the jumping loci of the factors, as follows.

Proposition 13.1. For $i \leq \min \left(\varkappa\left(X_{1}\right), \varkappa\left(X_{2}\right)\right)$,

(i) $\mathcal{V}_{1}^{i}\left(X_{1} \times X_{2}, \mathbb{k}\right)=\bigcup_{s+t=i} \mathcal{V}_{1}^{s}\left(X_{1}, \mathbb{k}\right) \times \mathcal{V}_{1}^{t}\left(X_{2}, \mathbb{k}\right)$

(ii) $\mathcal{R}_{1}^{i}\left(X_{1} \times X_{2}, \mathbb{k}\right)=\bigcup_{s+t=i} \mathcal{R}_{1}^{s}\left(X_{1}, \mathbb{k}\right) \times \mathcal{R}_{1}^{t}\left(X_{2}, \mathbb{k}\right)$.

Proof. (i) Write $X=X_{1} \times X_{2}$, and identify $G:=\pi_{1}(X)$ with $G_{1} \times G_{2}$. Let $\widetilde{X}=\widetilde{X}_{1} \times \widetilde{X}_{2}$ be the universal cover of $X$. We then have a $G$-equivariant isomorphism of chain complexes, $C_{\bullet}(\tilde{X}) \cong C_{\bullet}\left(\widetilde{X}_{1}\right) \otimes C_{\bullet}\left(\widetilde{X}_{2}\right)$. Given a character $\rho=\left(\rho_{1}, \rho_{2}\right)$ in $\operatorname{Hom}\left(G, \mathbb{k}^{\times}\right)=$ $\operatorname{Hom}\left(G_{1}, \mathbb{k}^{\times}\right) \times \operatorname{Hom}\left(G_{2}, \mathbb{k}^{\times}\right)$, we obtain an isomorphism $C_{\bullet}\left(X, \mathbb{k}_{\rho}\right) \cong C_{\bullet}\left(X_{1}, \mathbb{k}_{\rho_{1}}\right) \otimes_{\mathbb{k}}$ $C \cdot\left(X_{2}, \mathbb{k}_{\rho_{2}}\right)$. Taking homology, we find $H_{i}\left(X, \mathbb{k}_{\rho}\right)=\bigoplus_{s+t=i} H_{s}\left(X_{1}, \mathbb{k}_{\rho_{1}}\right) \otimes_{\mathbb{k}} H_{t}\left(X_{2}, \mathbb{k}_{\rho_{2}}\right)$, and the claim follows.

(ii) Write $A=H^{*}(X, \mathbb{k})$ and $A_{j}=H^{*}\left(X_{j}, \mathbb{k}\right)$, for $j=1,2$. By the Künneth formula, $A=A_{1} \otimes_{\mathbb{k}} A_{2}$. Pick an element $a=\left(a_{1}, a_{2}\right)$ in $A^{1}=A_{1}^{1} \oplus A_{2}^{1}$. The Aomoto complex of $X$ splits as a tensor product of chain complexes, $(A, a) \cong\left(A_{1}, a_{1}\right) \otimes_{\mathbb{k}}\left(A_{2}, a_{2}\right)$. The claim follows.

Various particular cases of this Proposition are known from the literature, see e.g. Corollary 3.3.2 in [32], Theorem 3.2 in [15], Lemma 7.5 in [22], and Proposition 5.7 in [35].

The next lemma shows that exponential tangent cones also behave well with respect to direct products; the proof is left as an exercise in the definitions.

Lemma 13.2. Let $G=G_{1} \times G_{2}$ be a product of finitely generated groups, and let $W_{1} \subseteq \mathbb{T}_{G_{1}}$ and $W_{2} \subseteq \mathbb{T}_{G_{2}}$ be Zariski closed subsets. Then, $\tau_{1}\left(W_{1} \times W_{2}\right)=\tau_{1}\left(W_{1}\right) \times \tau_{1}\left(W_{2}\right)$.

13.2. Jumping loci of wedges. We now express the jumping loci of the wedge $X_{1} \vee X_{2}$ in terms of the jumping loci of the factors.

Proposition 13.3. Suppose $b_{1}\left(X_{j}, \mathbb{k}\right) \neq 0$, for $j=1,2$. Then, for $i \leq \min \left(\varkappa\left(X_{1}\right), \varkappa\left(X_{2}\right)\right)$, 
(i) $\mathcal{V}_{1}^{i}\left(X_{1} \vee X_{2}, \mathbb{k}\right)= \begin{cases}\operatorname{Hom}\left(G_{1}, \mathbb{k}^{\times}\right) \times \operatorname{Hom}\left(G_{2}, \mathbb{k}^{\times}\right) & \text {if } i=1, \\ \mathcal{V}_{1}^{i}\left(X_{1}, \mathbb{k}\right) \times \operatorname{Hom}\left(G_{2}, \mathbb{k}^{\times}\right) \cup \operatorname{Hom}\left(G_{1}, \mathbb{k}^{\times}\right) \times \mathcal{V}_{1}^{i}\left(X_{2}, \mathbb{k}\right) & \text { if } i>1 .\end{cases}$
(ii) $\mathcal{R}_{1}^{i}\left(X_{1} \vee X_{2}, \mathbb{k}\right)= \begin{cases}H^{1}\left(X_{1}, \mathbb{k}\right) \times H^{1}\left(X_{2}, \mathbb{k}\right) & \text { if } i=1, \\ \mathcal{R}_{1}^{i}\left(X_{1}, \mathbb{k}\right) \times H^{1}\left(X_{2}, \mathbb{k}\right) \cup H^{1}\left(X_{1}, \mathbb{k}\right) \times \mathcal{R}_{1}^{i}\left(X_{2}, \mathbb{k}\right) & \text { if } i>1 .\end{cases}$

Proof. (i) Write $X=X_{1} \vee X_{2}$, and identify $G:=\pi_{1}(X)$ with $G_{1} * G_{2}$. Given a character $\rho=$ $\left(\rho_{1}, \rho_{2}\right)$ in $\operatorname{Hom}\left(G, \mathbb{k}^{\times}\right)=\operatorname{Hom}\left(G_{1}, \mathbb{k}^{\times}\right) \times \operatorname{Hom}\left(G_{2}, \mathbb{k}^{\times}\right)$, we have an isomorphism of chain complexes, $C_{+}\left(X, \mathbb{k}_{\rho}\right) \cong C_{+}\left(X_{1}, \mathbb{k}_{\rho_{1}}\right) \oplus C_{+}\left(X_{2}, \mathbb{k}_{\rho_{2}}\right)$. Write $b_{i}(X, \rho):=\operatorname{dim}_{\mathbb{k}} H_{i}\left(X, \mathbb{k}_{\rho}\right)$. We then have:

$$
b_{i}(X, \rho)= \begin{cases}b_{i}\left(X_{1}, \rho_{1}\right)+b_{i}\left(X_{2}, \rho_{2}\right)+1 & \text { if } i=1, \text { and } \rho_{1} \neq 1, \rho_{2} \neq 1 \\ b_{i}\left(X_{1}, \rho_{1}\right)+b_{i}\left(X_{2}, \rho_{2}\right) & \text { otherwise. }\end{cases}
$$

Since $b_{1}\left(X_{j}, 1\right)=b_{1}\left(X_{j}\right)>0$, the claim follows.

(ii) With notation as before, pick an element $a=\left(a_{1}, a_{2}\right)$ in $A^{1}=A_{1}^{1} \oplus A_{2}^{1}$. The Aomoto complex of $X$ splits (in positive degrees) as a direct sum of chain complexes, $\left(A^{+}, a\right) \cong\left(A_{1}^{+}, a_{1}\right) \oplus\left(A_{2}^{+}, a_{2}\right)$. We then have:

$$
\beta_{i}(A, a)= \begin{cases}\beta_{i}\left(A_{1}, a_{1}\right)+\beta_{i}\left(A_{2}, a_{2}\right)+1 & \text { if } i=1, \text { and } a_{1} \neq 0, a_{2} \neq 0, \\ \beta_{i}\left(A_{1}, a_{1}\right)+\beta_{i}\left(A_{2}, a_{2}\right) & \text { otherwise. }\end{cases}
$$

The claim follows.

Various particular cases of this Proposition are known from the literature, see e.g. Proposition 3.2.1 in [32], Corollary 5.4 in [44], and Lemma 7.8 in [22].

13.3. $\Sigma$-invariants of products and coproducts. The following product formula for the BNSR invariants was recently established by Bieri and Geoghegan in [8], building on work on several others. Their result, slightly modified to fit our setup, reads as follows.

Theorem 13.4 ([8]). Let $G_{1}$ and $G_{2}$ be two groups of type $\mathrm{F}_{k}(k \geq 1)$. For all $q \leq k$, the following hold:

(i) $\Sigma^{q}\left(G_{1} \times G_{2}, \mathbb{Z}\right)^{\complement} \subseteq \bigcup_{i+j=q} \Sigma^{i}\left(G_{1}, \mathbb{Z}\right)^{\complement} \times \Sigma^{j}\left(G_{2}, \mathbb{Z}\right)^{\complement}$, with equality for $q \leq 3$.

(ii) $\Sigma^{q}\left(G_{1} \times G_{2}, \mathbb{k}\right)^{\complement}=\bigcup_{i+j=q} \Sigma^{i}\left(G_{1}, \mathbb{k}\right)^{\complement} \times \Sigma^{j}\left(G_{2}, \mathbb{k}\right)^{\complement}$, for all fields $\mathbb{k}$.

The above product formula was established for $q=1$ by Bieri, Neumann, and Strebel in [10, Theorem 7.4], and was conjectured to hold in general (over $\mathbb{Z}$ ) by Bieri [6]. The inclusion from (i) was established by Meinert (unpublished), with a proof given by Gehrke [31] (see also Bieri [6]). The fact that equality holds in (i) for $q \leq 3$ was proved by Schütz [49] and Bieri-Geoghegan [8]. For $q \geq 4$, equality may fail in (i), as examples from [49] show.

As is well-known, the $\Sigma$-invariants of a non-trivial free product are all empty. For completeness, we include a proof of this fact.

Proposition 13.5. Let $G_{1}$ and $G_{2}$ be two non-trivial, finitely generated groups. Then $\Sigma^{q}\left(G_{1} * G_{2}, \mathbb{Z}\right)=\emptyset$, for all $q \geq 1$. 
Proof. This follows at once from a result of Bieri and Strebel, as recounted in [38, Lemma 2.4]: If $G=G_{1} *_{K} G_{2}$ is a non-trivial free product with amalgamation, and $\chi: G \rightarrow \mathbb{R}$ is a homomorphism such that $\left.\chi\right|_{K}=0$, then $\chi \notin \Sigma^{1}(G)$. In our situation, $K=\{1\}$; thus, $\Sigma^{1}(G)=\emptyset$, and so $\Sigma^{q}(G, \mathbb{Z})=\emptyset$, for all $q \geq 1$.

13.4. Upper bounds for products and coproducts. We now analyze the extent to which our bounds for the $\Sigma$-invariants carry over under direct products. We start by showing that, if the exponential tangent cone upper bound (29) holds as an equality for two groups, up to some degree $q$, then it also holds as an equality for their product, in degree $q$.

Theorem 13.6. Let $G_{1}$ and $G_{2}$ be two groups of type $\mathrm{F}_{k}(k \geq 1)$. For each $q \leq k$, the following holds:

$$
\begin{aligned}
\Sigma^{r}\left(G_{j}, \mathbb{Z}\right)=\left(\tau_{1}^{\mathbb{R}}\left(\bigcup_{i \leq r} \mathcal{V}_{1}^{i}\left(G_{j}, \mathbb{C}\right)\right)\right)^{\complement}, \quad \text { for } j=1,2 \text { and } r \leq q \\
\Longrightarrow \Sigma^{q}\left(G_{1} \times G_{2}, \mathbb{Z}\right)=\left(\tau_{1}^{\mathbb{R}}\left(\bigcup_{i \leq q} \mathcal{V}_{1}^{i}\left(G_{1} \times G_{2}, \mathbb{C}\right)\right)\right)^{\complement}
\end{aligned}
$$

Proof. Follows from Theorem 9.2, Proposition 13.1(i), Lemma 13.2, and Theorem 13.4(i).

Remark 13.7. As a byproduct of the proof of Theorem 13.6, we have the following: If the exponential tangent cone upper bound (29) holds as an equality for $G_{1}$ and $G_{2}$, up to degree $q$, then the Bieri Conjecture is true integrally for $G_{1} \times G_{2}$ in degree $q$, i.e., equality holds in Theorem 13.4(i).

As for (non-trivial) free products, we have the following observation.

Proposition 13.8. Let $G=G_{1} * G_{2}$ be a non-trivial free product of finitely generated groups. Then:

(i) The resonance upper bound (31) holds for $G$, in all degrees $q \geq 0$.

(ii) If $b_{1}\left(G_{1}\right)>0$ and $b_{1}\left(G_{2}\right)>0$, then the resonance upper bound (31) holds as an equality for $G$, in all degrees $q \geq 0$.

Proof. Follows from Propositions 13.5 and 13.3(ii).

\section{Toric COMPlexes And Right-Angled ARTin groups}

We now consider certain CW-complexes which interpolate between a wedge and a product of circles, and find that the "resonance upper bound" for the BNSR invariants holds for such spaces (and their fundamental groups), often with equality.

14.1. Toric complexes and Stanley-Reisner ring. Every finite simplicial complex $L$ gives rise to a finite $\mathrm{CW}$-complex, $T_{L}$. If $\mathrm{V}$ is the vertex set of $L$, and $T^{n}$ is the torus of dimension $n=|\mathrm{V}|$, with the standard CW-decomposition, then the toric complex $T_{L}$ is the subcomplex of $T^{n}$ obtained by deleting the cells corresponding to the non-faces of $L$.

The fundamental group, $G_{L}=\pi_{1}\left(T_{L}\right)$, coincides with the right-angled Artin group $G_{\Gamma}$ defined by the graph $\Gamma=L^{(1)}$. The group $G_{\Gamma}$ has a presentation consisting of a generator 
$v$ for each vertex $v \in \mathrm{V}$, and a commutator relation $v w=w v$ for each edge $\{v, w\}$ in $\Gamma$. A classifying space for $G_{\Gamma}$ is the toric complex $T_{\Delta}$, where $\Delta=\Delta_{\Gamma}$ is the flag complex of $\Gamma$. In particular, every right-angled Artin group is of type $\mathrm{F}$.

Since $T_{L}$ is a subcomplex of the $n$-torus, $H_{*}\left(T_{L}, \mathbb{Z}\right)$ is torsion-free. The cohomology ring of $T_{L}$, with coefficients in a field $\mathbb{k}$, is isomorphic to the exterior Stanley-Reisner $\operatorname{ring} \mathbb{k}\langle L\rangle$, with generators the duals $v^{*}$, and relations the monomials corresponding to the missing faces of $L$.

Given an element $z \in \mathbb{k}\langle L\rangle^{1}$, write $z=\sum_{v \in \mathrm{V}} z_{v} v^{*}$, with $z_{v} \in \mathbb{k}$, and define its support as $\operatorname{supp}(z)=\left\{v \in \mathrm{V} \mid z_{v} \neq 0\right\}$. For a subset $\mathrm{W} \subseteq \mathrm{V}$, write $z_{\mathrm{W}}=\sum_{v \in \mathrm{W}} v^{*}$. The calculation of the Aomoto-Betti numbers $\beta_{i}(\mathbb{k}\langle L\rangle, z)$ is greatly simplified by the following lemma.

Lemma 14.1 ([1]). Let $z \in \mathbb{k}\langle L\rangle^{1}$, and $\mathbf{W}=\operatorname{supp}(z)$. Then $\beta_{i}(\mathbb{k}\langle L\rangle, z)=\beta_{i}(\mathbb{k}\langle L\rangle$, zW $)$, for all $i \geq 0$.

The Aomoto-Betti numbers of the Stanley-Reisner ring may be computed solely in terms of the simplicial complex $L$, by means of the following formula from Aramova, Avramov, and Herzog [1], as slightly modified in [46]:

$$
\beta_{i}\left(\mathbb{k}\langle L\rangle, z_{\mathrm{W}}\right)=\sum_{\sigma \in L_{\bigvee \backslash W}} \operatorname{dim}_{\mathbb{k}} \widetilde{H}_{i-1-|\sigma|}\left(\mathrm{lk}_{L_{\mathrm{W}}}(\sigma), \mathbb{k}\right) .
$$

Here $L_{\mathrm{W}}=\{\tau \in L \mid \tau \subseteq \mathrm{W}\}$ is the induced subcomplex of $L$ on vertex set $\mathrm{W}$, and $\operatorname{lk}_{K}(\sigma)=$ $\{\tau \in K \mid \tau \cup \sigma \in L\}$ is the link in a subcomplex $K$ of a simplex $\sigma$.

14.2. Jumping loci of toric complexes. The resonance and characteristic varieties of toric complexes were computed in [46], extending previous results from [44] and [22].

The cohomology group $H^{1}\left(T_{L}, \mathbb{k}\right)=\mathbb{k}\langle L\rangle^{1}$ may be identified with the $\mathbb{k}$-vector space with basis $\mathrm{V}$, denoted $\mathbb{k}^{\mathrm{V}}$. For a subset $\mathrm{W} \subseteq \mathrm{V}$, let $\mathbb{k}^{\mathrm{W}}$ be the corresponding coordinate subspace. The character variety of the associated right-angled $\operatorname{Artin} \operatorname{group}, \operatorname{Hom}\left(G_{L}, \mathbb{k}^{\times}\right)$, may be identified with the algebraic torus $\left(\mathbb{k}^{\times}\right)^{\mathrm{V}}$. For a subset $\mathrm{W} \subseteq \mathrm{V}$, let $\left(\mathbb{k}^{\times}\right)^{\mathrm{W}}$ be the corresponding coordinate subtorus.

Theorem 14.2 ([46]). Let $L$ be a finite simplicial complex on vertex set $\mathrm{V}$, and let $T_{L}$ be the associated toric complex. Then, for any field $\mathbb{k}$, and every $i \geq 0$ and $d>0$,

(i) $\mathcal{V}_{d}^{i}\left(T_{L}, \mathbb{k}\right)=\bigcup_{\mathrm{W}}\left(\mathbb{k}^{\times}\right)^{\mathrm{W}}$,

(ii) $\mathcal{R}_{d}^{i}\left(T_{L}, \mathbb{k}\right)=\bigcup_{\mathrm{W}} \mathbb{k}^{\mathrm{W}}$,

where in both cases, the union is taken over all subsets $\mathrm{W} \subseteq \mathrm{V}$ for which $\beta_{i}(\mathbb{k}\langle L\rangle, z \mathrm{~W}) \geq d$.

Identify the exponential map $\operatorname{Hom}\left(G_{L}, \mathbb{C}\right) \rightarrow \operatorname{Hom}\left(G_{L}, \mathbb{C}^{\times}\right)$with the component-wise exponential $\mathbb{C}^{\mathrm{V}} \rightarrow\left(\mathbb{C}^{\times}\right)^{\mathrm{V}}$. Clearly, $\exp \left(\mathbb{C}^{\mathrm{W}}\right)=\left(\mathbb{C}^{\times}\right)^{\mathrm{W}}$, for all $\mathrm{W} \subseteq \mathrm{V}$.

Corollary 14.3. For each $i \geq 0$ and $d>0$,

(i) $\exp :\left(\mathcal{R}_{d}^{i}\left(T_{L}, \mathbb{C}\right), 0\right) \stackrel{\simeq}{\longrightarrow}\left(\mathcal{V}_{d}^{i}\left(T_{L}, \mathbb{C}\right), 1\right)$.

(ii) $\tau_{1}\left(\mathcal{V}_{d}^{i}\left(T_{L}, \mathbb{C}\right)\right)=T C_{1}\left(\mathcal{V}_{d}^{i}\left(T_{L}, \mathbb{C}\right)\right)=\mathcal{R}_{d}^{i}\left(T_{L}, \mathbb{C}\right)$.

(iii) $\Sigma^{q}\left(T_{L}, \mathbb{Z}\right) \subseteq\left(\bigcup_{i \leq q} \mathcal{R}_{1}^{i}\left(T_{L}, \mathbb{R}\right)\right)^{\complement}$, for all $q \geq 0$.

Proof. Part (i) follows from Theorem 14.2, Part (ii) is a consequence of Proposition 5.4, and Part (iii) is implied by Corollary 9.4 . 
14.3. $\Sigma$-invariants of right-angled Artin groups. The Bieri-Neumann-Strebel-Renz invariants of a right-angled Artin group $G_{\Gamma}$ were computed by Meier, Meinert, and VanWyk in [39]. A more convenient - yet equivalent - description of these invariants was given by Bux and Gonzalez in [13].

Theorem 14.4 ([39], [13]). Let $\mathbb{k}$ be a commutative ring. Let $\chi: G_{\Gamma} \rightarrow \mathbb{R}$ be a non-zero homomorphism, with support $\mathrm{W}=\{v \in \mathrm{V} \mid \chi(v) \neq 0\}$. Then, $\chi \in \Sigma^{q}\left(G_{\Gamma}, \mathbb{k}\right)$ if and only if $\widetilde{H}_{j}\left(\mathrm{lk}_{L_{\mathrm{W}}}(\sigma), \mathbb{k}\right)=0$, for every simplex $\sigma \in L_{\mathrm{V} \backslash \mathrm{W}}$, and every $-1 \leq j \leq q-\operatorname{dim}(\sigma)-2$.

Comparing the above description of $\Sigma^{q}\left(G_{\Gamma}, \mathbb{k}\right)$ to that of $\mathcal{R}_{1}^{i}\left(G_{\Gamma}, \mathbb{k}\right)$ from Theorem 14.2, and making use of formula (35), we obtain the following result.

Corollary 14.5. Let $\chi \in H^{1}\left(G_{\Gamma}, \mathbb{R}\right) \backslash\{0\}$. For any field $\mathbb{k}$, and any integer $q \geq 0$,

$$
\chi \in \Sigma^{q}\left(G_{\Gamma}, \mathbb{k}\right) \Longleftrightarrow z_{\operatorname{supp}(\chi)} \notin \bigcup_{i \leq q} \mathcal{R}_{1}^{i}\left(G_{\Gamma}, \mathbb{k}\right) .
$$

For $\mathbb{k}=\mathbb{R}$, we obtain the following corollary, showing that the "resonance upper bound" predicted by Corollary 9.4 is attained for right-angled Artin groups, at the price of replacing $\mathbb{Z}$-coefficients by $\mathbb{R}$-coefficients.

Corollary 14.6. $\Sigma^{q}\left(G_{\Gamma}, \mathbb{R}\right)=\left(\bigcup_{i \leq q} \mathcal{R}_{1}^{i}\left(G_{\Gamma}, \mathbb{R}\right)\right)^{\complement}$, for all $q \geq 0$.

Proof. Let $\Delta=\Delta_{\Gamma}$ be the flag complex of $\Gamma$, and recall that $T_{\Delta}=K\left(G_{\Gamma}, 1\right)$. By Lemma 14.1, we have $\beta_{i}(\mathbb{R}\langle\Delta\rangle, \chi)=\beta_{i}\left(\mathbb{R}\langle\Delta\rangle, z_{\operatorname{supp}(\chi)}\right)$. Thus, $\chi \in \mathcal{R}_{1}^{i}\left(G_{\Gamma}, \mathbb{R}\right)$ if and only if $z_{\operatorname{supp}(\chi)} \in \mathcal{R}_{1}^{i}\left(G_{\Gamma}, \mathbb{R}\right)$. The conclusion now follows from Corollary 14.5.

When $q=1$, this Corollary recovers Proposition 5.8 from [44], which says that $\Sigma^{1}\left(G_{\Gamma}\right)=$ $\mathcal{R}_{1}^{1}\left(G_{\Gamma}, \mathbb{R}\right)^{\complement}$. After adding appropriate conditions on the flag complex $\Delta=\Delta_{\Gamma}$, the resonance upper bound becomes an equality for all $q$, even integrally.

Corollary 14.7. Suppose that, for every simplex $\sigma \in \Delta$ and for every $\mathrm{W} \subseteq \mathrm{V}$ such that $\sigma \cap W=\emptyset$, the subcomplex $\mathrm{lk}_{\Delta_{W}}(\sigma)$ has torsion-free integral homology (this happens, for instance, if $\Gamma$ is a tree). Then:

$$
\Sigma^{q}\left(G_{\Gamma}, \mathbb{Z}\right)=\Sigma^{q}\left(G_{\Gamma}, \mathbb{k}\right)=\left(\bigcup_{i \leq q} \mathcal{R}_{1}^{i}\left(G_{\Gamma}, \mathbb{R}\right)\right)^{\complement},
$$

for all $q \geq 0$, and all fields $\mathbb{k}$.

The torsion-freeness condition in the above Corollary is really necessary, as shown in the next example.

Example 14.8. Let $\Delta$ be a flag triangulation of the real projective plane, $\mathbb{R P}^{2}$, and let $\nu: G_{\Delta} \rightarrow \mathbb{Z}$ be the diagonal homomorphism, sending each standard generator to 1 . Then $\nu \notin \Sigma^{2}\left(G_{\Delta}, \mathbb{Z}\right)$ and $\nu \notin \Sigma^{2}\left(G_{\Delta}, \mathbb{k}\right)$, if char $\mathbb{k}=2$, but $\nu \in \Sigma^{2}\left(G_{\Delta}, \mathbb{k}\right)$, if char $\mathbb{k} \neq 2$. In particular, $\nu \in \Sigma^{2}\left(G_{\Delta}, \mathbb{R}\right) \backslash \Sigma^{2}\left(G_{\Delta}, \mathbb{Z}\right)$.

\section{Free abelian covers of toric COMplexes And Artin kernels}

We now study the homological finiteness properties of the free abelian Galois covers of a toric complex. In the case of cyclic covers, we also study the jumping loci and the BNS invariants of the corresponding "Artin kernels." 
15.1. Homological finiteness of toric covers. We start with an elementary lemma.

Lemma 15.1. Let $\nu: \mathbb{Z}^{n} \rightarrow \mathbb{Z}^{r}$ and $\mu: \mathbb{Z}^{n} \rightarrow \mathbb{Z}^{s}$ be two epimorphisms, and let $\mathbb{k}$ be an infinite field. The following are equivalent:

(1) $\nu^{*}\left(\operatorname{Hom}\left(\mathbb{Z}^{r}, \mathbb{k}^{\times}\right)\right) \cap \mu^{*}\left(\operatorname{Hom}\left(\mathbb{Z}^{s}, \mathbb{k}^{\times}\right)\right)$is finite.

(2) $\nu^{*}\left(H^{1}\left(\mathbb{Z}^{r}, \mathbb{Q}\right)\right) \cap \mu^{*}\left(H^{1}\left(\mathbb{Z}^{s}, \mathbb{Q}\right)\right)=\{0\}$.

Proof. Write $A=\operatorname{ker}(\nu)$ and $B=\operatorname{ker}(\mu)$. Then,

$$
\nu^{*}\left(\operatorname{Hom}\left(\mathbb{Z}^{r}, \mathbb{k}^{\times}\right)\right) \cap \mu^{*}\left(\operatorname{Hom}\left(\mathbb{Z}^{s}, \mathbb{k}^{\times}\right)\right)=\operatorname{Hom}\left(\mathbb{Z}^{n} /(A+B), \mathbb{k}^{\times}\right)
$$

is finite if and only if the group $\mathbb{Z}^{n} /(A+B)$ is finite (since $\mathbb{k}$ is infinite). In turn, this is equivalent to $\operatorname{Hom}\left(\mathbb{Z}^{n} /(A+B), \mathbb{Q}\right)=\{0\}$, or $\nu^{*}\left(\operatorname{Hom}\left(\mathbb{Z}^{r}, \mathbb{Q}\right)\right) \cap \mu^{*}\left(\operatorname{Hom}\left(\mathbb{Z}^{s}, \mathbb{Q}\right)\right)=\{0\}$.

Now let $L$ be a simplicial complex on vertex set $\mathrm{V}$, with $n=|\mathrm{V}|$.

Theorem 15.2. Let $\nu: G_{L} \rightarrow \mathbb{Z}^{r}$ be an epimorphism, $\mathbb{k}$ a field, and $\nu^{*}: H^{1}\left(\mathbb{Z}^{r}, \mathbb{Q}\right) \rightarrow$ $H^{1}\left(G_{L}, \mathbb{Q}\right)$ the induced homomorphism. For each $q \geq 0$, the following are equivalent:

(i) $\operatorname{dim}_{\mathbb{k}} H_{i}\left(T_{L}^{\nu}, \mathbb{k}\right)<\infty$, for all $i \leq q$.

(ii) $\operatorname{im}\left(\nu^{*}\right) \cap \mathbb{Q}^{\mathrm{W}}=\{0\}$, for all $\mathrm{W} \subseteq \mathrm{V}$ such that $\beta_{i}\left(\mathbb{k}\langle L\rangle, z_{\mathrm{W}}\right) \neq 0$, for some $i \leq q$.

Proof. Without loss of generality, we may assume $\mathbb{k}=\overline{\mathbb{k}}$. By Corollary 6.2 and Theorem 14.2(i), the dimension of $H_{\leq q}\left(T_{L}^{\nu}, \mathbb{k}\right)$ is finite if and only if the set

$$
\nu^{*}\left(\operatorname{Hom}\left(\mathbb{Z}^{r}, \mathbb{k}^{\times}\right)\right) \cap\left(\bigcup_{i \leq q} \bigcup_{\substack{W \subseteq V \\ \beta_{i}(\mathbb{K}\langle L\rangle, z \mathrm{~W}) \neq 0}}\left(\mathbb{k}^{\times}\right)^{\mathrm{W}}\right)
$$

is finite. Each coordinate subtorus $\left(\mathbb{R}^{\times}\right)^{\mathrm{W}}$ may be viewed as the pullback of $\operatorname{Hom}\left(\mathbb{Z}^{s}, \mathbb{k}^{\times}\right)$ via a suitable epimorphism $\mu: \mathbb{Z}^{n} \rightarrow \mathbb{Z}^{s}$; in this case, we also have $\mathbb{Q}^{\mathrm{W}}=\mu^{*}\left(H^{1}\left(\mathbb{Z}^{s}, \mathbb{Q}\right)\right)$. The claim now follows from Lemma 15.1.

In view of Theorem 15.2 and formula (35), the problem of deciding whether the Betti numbers of $T_{L}^{\nu}$ (with coefficients in a field $\mathbb{k}$ ) are finite, up to a certain degree, reduces to a purely combinatorial and linear algebra question.

In characteristic 0, Theorem 15.2 together with Theorem 14.2(ii) yield the following corollary.

Corollary 15.3. For each $q \geq 0$,

$$
\operatorname{dim}_{\mathbb{Q}} H_{\leq q}\left(T_{L}^{\nu}, \mathbb{Q}\right)<\infty \Longleftrightarrow \nu^{*}\left(H^{1}\left(\mathbb{Z}^{r}, \mathbb{Q}\right)\right) \cap\left(\bigcup_{i \leq q} \mathcal{R}_{1}^{i}\left(T_{L}, \mathbb{Q}\right)\right)=\{0\} .
$$

Theorem 15.2 also leads to the following qualitative result.

Corollary 15.4. For all integers $r \geq 1$ and $q \geq 0$, and all coefficient fields $\mathbb{k}$, the set

$$
\left\{\nu: G_{L} \rightarrow \mathbb{Z}^{r} \mid \operatorname{dim}_{\mathbb{k}} H_{\leq q}\left(T_{L}^{\nu}, \mathbb{k}\right)<\infty\right\}
$$

is a Zariski open subset of $\operatorname{Gr}_{r}\left(H^{1}\left(T_{L}, \mathbb{Q}\right)\right)$.

When $r>1$, this result stands in marked contrast with the case of an arbitrary CWcomplex $X$; see Remark 6.9. 
15.2. Artin kernels. Consider now an epimorphism $\chi: G_{L} \rightarrow \mathbb{Z}$. We then have an infinite cyclic (regular) cover, $T_{L}^{\chi} \rightarrow T_{L}$. The fundamental group $N_{\chi}:=\pi_{1}\left(T_{L}^{\chi}\right)=\operatorname{ker}(\chi)$ is called the Artin kernel associated to $\chi$. Clearly, a classifying space for this group is the space $T_{\Delta}^{\chi}$, where $\Delta=\Delta_{\Gamma}$ is the flag complex of $\Gamma=L^{(1)}$.

The most basic example is provided by the "diagonal" homomorphism, $\nu: G_{L} \rightarrow \mathbb{Z}$, given by $\nu(v)=1$, for all $v \in \mathrm{V}$. The corresponding Artin kernel, $N_{\Gamma}:=N_{\nu}$, is called the Bestvina-Brady group associated to $\Gamma$. As shown in [4], the homological finiteness properties of the group $N_{\Gamma}$ are intimately connected to the connectivity properties of the flag complex $\Delta$. For example, $N_{\Gamma}$ is finitely generated if and only if $\Gamma$ is connected; and $N_{\Gamma}$ is finitely presented if and only if $\Delta$ is simply-connected.

In [45, Proposition 6.1], we showed that all finitely presented Bestvina-Brady groups are 1-formal. In Lemma 9.1, Theorem 10.1, and Corollary 10.2 from [46], we extended this result to all Artin kernels satisfying certain homological conditions, as follows.

Theorem $15.5([46])$. Let $\chi: G_{\Gamma} \rightarrow \mathbb{Z}$ be an epimorphism, and $N_{\chi}=\operatorname{ker}(\chi)$ the corresponding Artin kernel.

(i) If $H_{1}\left(N_{\chi}, \mathbb{Q}\right)$ is a trivial $\mathbb{Q} \mathbb{Z}$-module, then $N_{\chi}$ is finitely generated.

(ii) If both $H_{1}\left(N_{\chi}, \mathbb{Q}\right)$ and $H_{2}\left(N_{\chi}, \mathbb{Q}\right)$ have trivial $\mathbb{Q} \mathbb{Z}$-action, then $N_{\chi}$ is 1-formal.

In particular, if $\Gamma$ is a connected graph, and $H_{1}\left(\Delta_{\Gamma}, \mathbb{Q}\right)=0$, then $N_{\Gamma}$ is 1-formal.

When condition (i) is satisfied, we also showed in [46, Lemma 9.1] that the inclusion map $\iota: N_{\chi} \hookrightarrow G_{L}$ induces a monomorphism on abelianizations; in particular, $H_{1}\left(N_{\chi}, \mathbb{Z}\right)$ is free abelian (of rank $|\mathrm{V}|-1$ ).

As explained in [46, Theorem 6.2], the $\mathbb{Q} \mathbb{Z}$-triviality conditions from (i) and (ii) above can be tested combinatorially. For instance, when $\chi=\nu$ is the Bestvina-Brady character, $H_{1}\left(N_{\Gamma}, \mathbb{Q}\right)$ has trivial $\mathbb{Q} \mathbb{Z}$-action if and only if $\Gamma$ is connected, while $H_{\leq 2}\left(N_{\Gamma}, \mathbb{Q}\right)$ has trivial $\mathbb{Q} \mathbb{Z}$-action if and only if $\widetilde{H}_{\leq 1}\left(\Delta_{\Gamma}, \mathbb{Q}\right)=0$.

15.3. Jumping loci of Artin kernels. In [45, Lemma 8.3], we established a relationship between the degree 1 (co)homology jumping loci of a finitely presented Bestvina-Brady group, and the jumping loci of the corresponding right-angled Artin group. We now generalize this result to Artin kernels. To avoid trivialities, we will assume for the rest of this section that $|\mathrm{V}|>1$.

Theorem 15.6. Let $\chi: G_{L} \rightarrow \mathbb{Z}$ be an epimorphism, $N_{\chi}=\operatorname{ker}(\chi)$, and $\iota: N_{\chi} \hookrightarrow G_{L}$ the inclusion map.

(i) Suppose $H_{1}\left(N_{\chi}, \mathbb{Q}\right)$ has trivial $\mathbb{Q} \mathbb{Z}$-action, and $\mathbb{k}=\overline{\mathbb{k}}$. Then the induced homomorphism $\iota^{*}: \operatorname{Hom}\left(G_{L}, \mathbb{k}^{\times}\right) \rightarrow \operatorname{Hom}\left(N_{\chi}, \mathbb{k}^{\times}\right)$restricts to a surjection, $\iota^{*}: \mathcal{V}_{1}^{1}\left(G_{L}, \mathbb{k}\right) \rightarrow$ $\mathcal{V}_{1}^{1}\left(N_{\chi}, \mathbb{k}\right)$

(ii) Suppose $H_{i}\left(N_{\chi}, \mathbb{Q}\right)$ has trivial $\mathbb{Q} \mathbb{Z}$-action, for $i=1,2$. Then the induced homomorphism $\iota^{*}: \operatorname{Hom}\left(G_{L}, \mathbb{C}\right) \rightarrow \operatorname{Hom}\left(N_{\chi}, \mathbb{C}\right)$ restricts to a surjection, $\iota^{*}: \mathcal{R}_{1}^{1}\left(G_{L}, \mathbb{C}\right) \rightarrow$ $\mathcal{R}_{1}^{1}\left(N_{\chi}, \mathbb{C}\right)$.

Proof. Write $G=G_{L}$ and $N=N_{\chi}$. We know from Theorem 15.5(i) that $N$ is finitely generated. 
(i) By [46, Lemma 9.1], $N^{\prime}=G^{\prime}$; hence, $N^{\prime \prime}=G^{\prime \prime}$. Therefore, the map $\iota: N \hookrightarrow G$ induces an isomorphism $N^{\prime} / N^{\prime \prime} \stackrel{\simeq}{\longrightarrow} G^{\prime} / G^{\prime \prime}$ between the respective Alexander invariants. Taking supports (over $\mathbb{k}$ ), and using Corollary 3.7 finishes the proof.

(ii) By [46, Theorem 9.5], the map $\mathfrak{h}(N, \mathbb{C}) \rightarrow \mathfrak{h}(G, \mathbb{C})$ induced by $\iota$ on holonomy Lie algebras restricts to an isomorphism $\mathfrak{h}^{\prime}(N, \mathbb{C}) \stackrel{\simeq}{\longrightarrow} \mathfrak{h}^{\prime}(G, \mathbb{C})$. Abelianizing, we obtain an isomorphism $\mathfrak{h}^{\prime}(N, \mathbb{C}) / \mathfrak{h}^{\prime \prime}(N, \mathbb{C}) \stackrel{\simeq}{\longrightarrow} \mathfrak{h}^{\prime}(G, \mathbb{C}) / \mathfrak{h}^{\prime \prime}(G, \mathbb{C})$. Taking supports (over $\mathbb{C}$ ), and using Remark 5.2 finishes the proof.

Denote by $\mathrm{V}_{0}(\chi):=\{v \in \mathrm{V} \mid \chi(v) \neq 0\}$ the support of $\chi$. For a subset $\mathrm{W} \subset \mathrm{V}$, denote by $\Gamma_{\mathrm{W}}$ the subgraph induced by $\Gamma$ on $\mathrm{W}$.

Corollary 15.7. With notation as above,

(i) Suppose the module $H_{1}\left(N_{\chi}, \mathbb{Q}\right)$ is $\mathbb{Q} \mathbb{Z}$-trivial, and $\mathbb{k}=\overline{\mathbb{k}}$. Then $\mathcal{V}_{1}^{1}\left(N_{\chi}, \mathbb{k}\right)$ is a union of subtori of the algebraic torus $\left(\mathbb{k}^{\times}\right)^{\mathrm{V}}$ :

$$
\mathcal{V}_{1}^{1}\left(N_{\chi}, \mathbb{k}\right)=\bigcup_{\substack{W \subset V \\ \Gamma_{\mathrm{W}} \text { disconnected }}} \iota^{*}\left(\left(\mathbb{k}^{\times}\right)^{\mathrm{W}}\right) .
$$

If $\mathrm{V}_{0}(\chi) \subseteq \mathrm{W}$, then $\operatorname{dim} \iota^{*}\left(\left(\mathbb{k}^{\times}\right)^{\mathrm{W}}\right)=|\mathrm{W}|-1$; otherwise, $\operatorname{dim} \iota^{*}\left(\left(\mathbb{k}^{\times}\right)^{\mathrm{W}}\right)=|\mathrm{W}|$.

(ii) Suppose the module $H_{\leq 2}\left(N_{\chi}, \mathbb{Q}\right)$ is $\mathbb{Q} \mathbb{Z}$-trivial. Then $\mathcal{R}_{1}^{1}\left(N_{\chi}, \mathbb{C}\right)$ is a union of rationally defined linear subspaces of the form $\iota^{*}\left(\mathbb{C}^{\mathrm{W}}\right)$, with the union indexed as in Part (i), and the dimensions computed likewise.

Proof. Combine Theorems 15.6 and 14.2, and use formula (35).

15.4. The BNS invariant of an Artin kernel. We now give a cohomological "upper bound" for the Bieri-Neumann-Strebel invariant of $N_{\chi}$.

Theorem 15.8. Suppose $H_{1}\left(N_{\chi}, \mathbb{Q}\right)$ has trivial $\mathbb{Q} \mathbb{Z}$-action. Then

$$
\Sigma^{1}\left(N_{\chi}\right) \subseteq\left(\bigcup_{\mathrm{W}} \iota^{*}\left(\mathbb{R}^{\mathrm{W}}\right)\right)^{\mathrm{C}}
$$

where $\mathrm{W}$ runs through all subsets of $\mathrm{V}$ such that $\Gamma_{\mathrm{W}}$ is disconnected. If, in addition, $H_{2}\left(N_{\chi}, \mathbb{Q}\right)$ is $\mathbb{Q} \mathbb{Z}$-trivial, the upper bound coincides with $\mathcal{R}_{1}^{1}\left(N_{\chi}, \mathbb{R}\right)^{\complement}$.

Proof. By Theorem 15.5(i), the group $N_{\chi}$ is finitely generated. By Theorem 9.2, the set $\Sigma^{1}\left(N_{\chi}\right)$ is contained in the complement of $\tau_{1}^{\mathbb{R}}\left(\mathcal{V}_{1}^{1}\left(N_{\chi}, \mathbb{C}\right)\right)$. Using Proposition 4.4(ii) and Corollary 15.7(i), we find that

$$
\tau_{1}^{\mathbb{R}}\left(\mathcal{V}_{1}^{1}\left(N_{\chi}, \mathbb{C}\right)\right)=\bigcup_{\mathrm{W}} T C_{1}^{\mathbb{R}}\left(\iota^{*}\left(\left(\mathbb{C}^{\times}\right)^{\mathrm{W}}\right)\right)=\bigcup_{\mathrm{W}} \iota^{*}\left(\mathbb{R}^{\mathrm{W}}\right),
$$

and this verifies our first claim. The second claim follows from Corollary 15.7(ii).

Under suitable assumptions, the above upper bound may be improved to a precise computation of $\Sigma^{1}\left(N_{\chi}\right)$.

Corollary 15.9. Assume $H_{1}\left(N_{\chi}, \mathbb{Q}\right)$ is $\mathbb{Q} \mathbb{Z}$-trivial. Then $\bigcup_{\mathrm{W}} \iota^{*}\left(\mathbb{R}^{\mathrm{W}}\right)=H^{1}\left(N_{\chi}, \mathbb{R}\right)$ if and only if there is a cut vertex $v \in \mathrm{V}$ such that $\chi(v) \neq 0$. When this holds, $\Sigma^{1}\left(N_{\chi}\right)=\emptyset$. 
Proof. Since $H_{1}\left(N_{\chi}, \mathbb{Q}\right)$ is $\mathbb{Q} \mathbb{Z}$-trivial, Theorem 6.2 from [46] implies that the graph $\Gamma$ is connected. The first assertion is then an easy consequence of Corollary 15.7, while the second assertion follows from Theorem 15.8.

Corollary 15.10. If $\Gamma$ is connected, and has a cut vertex, then $\Sigma^{1}\left(N_{\Gamma}\right)=\emptyset$.

We conclude this section by constructing an example of a finitely generated (1-formal) group which admits no finite presentation, yet for which the BNS invariant can be computed exactly.

Example 15.11. Let $K$ be a flag triangulation of the real projective plane, $\mathbb{R P}^{2}$, and let $L=K \cup e$ be the simplicial complex obtained from $K$ by adding an edge $e=\{v, w\}$, with $v$ a vertex of $K$, and $w$ a new vertex. Clearly, $L$ is also a flag complex; write $\Gamma=L^{(1)}$, so that $L=\Delta_{\Gamma}$. The vertex $v$ is a cut point for the graph $\Gamma$. Let $\chi$ be the character of $G_{\Gamma}$ sending the vertices of $K$ to 1 , and the new vertex to $p$, where $p$ is 1 or a prime number.

By Theorem 6.2 from [46], the $\mathbb{Q} \mathbb{Z}$-module $H_{\leq 2}\left(N_{\chi}, \mathbb{Q}\right)$ is trivial. Thus, by Theorem 15.5, the group $N_{\chi}$ is finitely generated and 1-formal. On the other hand, $\pi_{1}(L) \neq 0$, and thus, by the Main Theorem of [39], the group $N_{\chi}$ is not finitely presentable. Finally, by Corollary $15.9, \Sigma^{1}\left(N_{\chi}\right)=\emptyset$.

\section{KäHLER AND QUASI-KÄHLER MANIFOLDS}

In this final section, we discuss the relationship between the $\Sigma$-invariants and the homology jumping loci of Kähler and quasi-Kähler manifolds and their fundamental groups.

16.1. Formality. A compact, connected, complex manifold $M$ is called a Kähler manifold if $M$ admits a Hermitian metric for which the imaginary part is a closed 2-form. A manifold $X$ is called quasi-Kähler if $X=M \backslash D$, where $M$ is a compact Kähler manifold and $D$ is a divisor with normal crossings.

The Hodge structure on $H^{*}(M, \mathbb{C})$ puts strong constraints on the topology of a compact Kähler manifold $M$. For example, a basic result of Deligne, Griffiths, Morgan, and Sullivan [17] guarantees that $M$ must be formal. Consequently, the fundamental group of $M$ must be 1-formal.

For a quasi-Kähler manifold $X$, the mixed Hodge structure $\left(W_{\bullet}, F^{\bullet}\right)$ on $H^{*}(X, \mathbb{C})$, as defined by Deligne [16], puts its own constraints on the topology of $X$. Of course, these constraints are weaker: for example, $\pi_{1}(X)$ need not be 1-formal. Nevertheless, if $W_{1}\left(H^{1}(X, \mathbb{C})\right)=0$, then $\pi_{1}(X)$ is 1-formal, as shown by Morgan [40]. This vanishing property holds whenever $X$ admits a non-singular compactification $\bar{X}$ with $b_{1}(\bar{X})=0$, cf. [16]. These two facts together establish the 1-formality of fundamental groups of complements of projective hypersurfaces.

16.2. Jumping loci. Foundational results on the structure of the cohomology support loci for local systems on smooth projective varieties, and more generally, on compact Kähler manifolds were obtained by Beauville, Green and Lazarsfeld, Simpson, and Campana. A more general result, valid in the quasi-Kähler case, was obtained by Arapura [2]. We recall this result, in a simplified form, which is all we need for our purposes here. 
Theorem 16.1 ([2]). Let $X$ be a quasi-Kähler manifold, with fundamental group $G=$ $\pi_{1}(X)$. Then, all the components of $\mathcal{V}_{d}^{i}(X, \mathbb{C})$ passing through the origin are subtori of $\mathbb{T}_{G}=\operatorname{Hom}\left(G, \mathbb{C}^{\times}\right)$, provided one of the following conditions holds.

(a) $i=d=1$.

(b) $X$ is Kähler.

(c) $W_{1}\left(H^{1}(X, \mathbb{C})\right)=0$.

Proposition 4.4(ii) implies that $\tau_{1}\left(\mathcal{V}_{d}^{i}(X, \mathbb{C})\right)=T C_{1}\left(\mathcal{V}_{d}^{i}(X, \mathbb{C})\right)$, whenever one of the above conditions is satisfied. Moreover, by the remarks in $\S 16.1$, the group $G$ is 1-formal, provided either (b) or (c) is satisfied. In this case, $T C_{1}\left(\mathcal{V}_{d}^{1}(G, \mathbb{C})\right)=\mathcal{R}_{d}^{1}(G, \mathbb{C})$, by [23].

16.3. $\Sigma$-invariants. Using Theorem 16.1 and our results from $\S 9$, we obtain the following upper bounds for the BNSR invariants of a quasi-Kähler manifold, and its fundamental group.

Theorem 16.2. Let $X$ be a quasi-Kähler manifold, and $G=\pi_{1}(X)$. Then

(i) $\Sigma^{1}(G) \subseteq T C_{1}^{\mathbb{R}}\left(\mathcal{V}_{1}^{1}(G, \mathbb{C})\right)^{\complement}$.

(ii) Suppose $X$ is Kähler, or $W_{1}\left(H^{1}(X, \mathbb{C})\right)=0$. Then $\mathcal{R}_{1}^{1}(G, \mathbb{R})$ is a finite union of rationally defined linear subspaces, and $\Sigma^{1}(G) \subseteq \mathcal{R}_{1}^{1}(G, \mathbb{R})^{\complement}$.

(iii) Suppose $X$ is Kähler, or $W_{1}\left(H^{1}(X, \mathbb{C})\right)=0$. Then $U:=T C_{1}^{\mathbb{R}}\left(\bigcup_{i \leq q} \mathcal{V}_{1}^{i}(X, \mathbb{C})\right)$ is a finite union of rationally defined linear subspaces, and $\Sigma^{q}(X, \mathbb{Z}) \subseteq U^{\mathrm{C}}$, for all $q \geq 0$.

Proof. Part (i) follows from Corollary 9.3 and Theorem 16.1, part (a).

Part (ii) follows from Proposition 11.1 and the formality of $G$.

Part (iii) follows from Corollary 9.3 and Theorem 16.1, part (b) or (c).

The assumptions from (ii) are really necessary, as illustrated by the next example.

Example 16.3. Let $X$ be the complex Heisenberg manifold, defined as the total space of the $\mathbb{C}^{\times}$bundle over $\left(\mathbb{C}^{\times}\right)^{2}$ with Euler number 1 . As noted in [40], $X$ is a smooth quasi-projective variety. Moreover, the fundamental group $G=\pi_{1}(X)$ is isomorphic to the Heisenberg group from Example 12.5. Thus, $\Sigma^{1}(G) \nsubseteq \mathcal{R}_{1}^{1}(G, \mathbb{R})^{\complement}$.

16.4. Pencils. Next, we determine precisely when the resonance upper bound for $\Sigma^{1}$ is attained in the case when $M$ is a compact Kähler manifold. The answer is in terms of pencils, that is, holomorphic maps from $M$ onto smooth complex curves, having connected generic fibers.

We shall need a result of Arapura (contained in the proof of Proposition V.1.7 from [2]): If $f: M \rightarrow C$ is a pencil with $\chi(C) \leq 0$, then $H_{1}\left(M, \mathbb{C}_{f^{*} \rho}\right) \cong H_{1}\left(C, \mathbb{C}_{\rho}\right)$, for all but finitely many local systems $\rho \in \mathbb{T}_{\pi_{1}(C)}$.

Theorem 16.4. Let $M$ be a compact Kähler manifold with $b_{1}(M)>0$, and $G=\pi_{1}(M)$. The following are equivalent:

(i) $\Sigma^{1}(G)=\mathcal{R}_{1}^{1}(G, \mathbb{R})^{\complement}$.

(ii) If $f: M \rightarrow C$ is an elliptic pencil (i.e., if $\chi(C)=0$ ), then $f$ has no fibers with multiplicity greater than 1 . 
Proof. By a recent theorem of Delzant [19],

$$
\Sigma^{1}(G)^{\complement}=\bigcup f_{\alpha}^{*}\left(H^{1}\left(C_{\alpha}, \mathbb{R}\right)\right)
$$

where the union is taken over those pencils $f_{\alpha}: M \rightarrow C_{\alpha}$ with the property that either $\chi\left(C_{\alpha}\right)<0$, or $\chi\left(C_{\alpha}\right)=0$ and $f_{\alpha}$ has some multiple fiber. On the other hand, Theorem $\mathrm{B}$ from [22] together with Proposition V.1.7 from [2] imply that

$$
\mathcal{R}_{1}^{1}(G, \mathbb{R})=\bigcup f_{\beta}^{*}\left(H^{1}\left(C_{\beta}, \mathbb{R}\right)\right),
$$

where the union is taken over those pencils $f_{\beta}: M \rightarrow C_{\beta}$ with $\chi\left(C_{\beta}\right)<0$. To complete the proof, then, we only need to check that $f^{*}\left(H^{1}(C, \mathbb{R})\right) \nsubseteq \mathcal{R}_{1}^{1}(G, \mathbb{R})$, whenever $f: M \rightarrow C$ is an elliptic pencil.

An easy computation shows that $H_{1}\left(C, \mathbb{C}_{\rho}\right)=0$, for all non-trivial characters $\rho \in \mathbb{T}_{\pi_{1}(C)}$. Now suppose $f_{\beta}: M \rightarrow C_{\beta}$ is a pencil with $\chi\left(C_{\beta}\right)<0$. Another computation shows that $H_{1}\left(C_{\beta}, \mathbb{C}_{\rho}\right) \neq 0$, for all $\rho \in \mathbb{T}_{\pi_{1}\left(C_{\beta}\right)}$. By the above result of Arapura, $f^{*}\left(\mathbb{T}_{\pi_{1}(C)}\right) \nsubseteq$ $f_{\beta}^{*}\left(\mathbb{T}_{\pi_{1}\left(C_{\beta}\right)}\right)$. Using (38), we conclude that $f^{*}\left(H^{1}(C, \mathbb{R})\right) \nsubseteq \mathcal{R}_{1}^{1}(G, \mathbb{R})$.

Example 16.5. Let $C$ be a Riemann surface of genus $g \geq 1$, and $G=\pi_{1}(C)$. We then have, for any field $\mathbb{k}$ :

(i) $\mathcal{V}_{d}^{1}(G, \mathbb{k})=\left(\mathbb{k}^{\times}\right)^{2 g}$ for $d<2 g-1$, and $\mathcal{V}_{2 g-1}^{1}(G, \mathbb{k})=\{1\}$.

(ii) $\mathcal{R}_{d}^{1}(G, \mathbb{k})=\mathbb{k}^{2 g}$ for $d<2 g-1$, and $\mathcal{R}_{2 g-1}^{1}(G, \mathbb{k})=\{0\}$.

(iii) $\Sigma^{q}(G, \mathbb{Z})=\emptyset$, for all $q \geq 1$, if $g>1$, and $\Sigma^{q}(G, \mathbb{Z})^{\complement}=\{0\}$, for all $q$, if $g=1$.

In particular, the resonance upper bound for $\Sigma^{1}$ is attained for all non-trivial surface groups.

Remark 16.6. The equality $\Sigma^{1}(G)=\mathcal{R}_{1}^{1}(G, \mathbb{R})^{\complement}$ does not hold for arbitrary Kähler groups. Indeed, there is a classical way of constructing elliptic pencils with multiple fibers on compact Kähler manifolds, starting from convenient finite group actions; see Beauville [3, Example 1.8].

16.5. Hyperplane arrangements. Among complex hypersurfaces, probably the best understood are the unions of hyperplanes. Let $\mathcal{A}$ be a finite collection of hyperplanes in $\mathbb{C}^{\ell}$, with union $V=\bigcup_{H \in \mathcal{A}} H$. The complement of the arrangement, $X=\mathbb{C}^{\ell} \backslash V$, is a formal space.

The homology groups $H_{*}(X, \mathbb{Z})$ are torsion-free. The cohomology ring $A=H^{*}(X, \mathbb{Z})$ depends only on the combinatorics of the arrangement, as encoded in the intersection lattice, $L(\mathcal{A})$. Thus, the resonance varieties, $\mathcal{R}_{d}^{i}(X, \mathbb{k})$, are also combinatorially determined, for any field $\mathbb{k}$.

It follows from the work of Esnault, Schechtman, and Viehweg [26] that the exponential formula (17) holds for any arrangement complement $X$, for all $i \geq 0$ and $d>0$. By Proposition 5.4, the resonance varieties $\mathcal{R}_{d}^{i}(X, \mathbb{C})$ are finite unions of rationally defined linear subspaces.

As an application of Corollary 9.4, we obtain the following combinatorial upper bounds for the BNSR invariants of arrangements.

Proposition 16.7. Let $\mathcal{A}$ be a hyperplane arrangement in $\mathbb{C}^{\ell}$, with complement $X$. Then $\Sigma^{q}(X, \mathbb{Z}) \subseteq\left(\bigcup_{i \leq q} \mathcal{R}_{1}^{i}(X, \mathbb{R})\right)^{\complement}$, for all $q \geq 0$. 
It would be interesting to know whether the equality $\Sigma^{1}(G)=\mathcal{R}_{1}^{1}(G, \mathbb{R})^{\complement}$ holds for arrangement groups, $G=\pi_{1}(X)$. For a complexified real arrangement, it is easy to see that complex conjugation in $\mathbb{C}^{\ell}$ restricts to a homeomorphism, $\alpha: X \stackrel{\simeq}{\longrightarrow} X$, with the property that $\alpha_{*}=-\mathrm{id}$ on $H_{1}(X, \mathbb{Z})$. Thus, $\Sigma^{1}(G)=-\Sigma^{1}(G)$, which is consistent with the symmetry property of $\mathcal{R}_{1}^{1}(G, \mathbb{R})^{\complement}$.

Acknowledgments. A substantial part of this work was carried out while the second author was visiting the Institute of Mathematics of the Romanian Academy in October 2007, and again in July 2008. He thanks the Institute for its support and hospitality during his stays in Bucharest, Romaniageferences

[1] A. Aramova, L. Avramov, J. Herzog, Resolutions of monomial ideals and cohomology over exterior algebras, Trans. Amer. Math. Soc. 352 (1999), no. 2, 579-594. MR1603874 14.1

[2] D. Arapura, Geometry of cohomology support loci for local systems I, J. Alg. Geometry 6 (1997), no. 3, 563-597. MR1487227 1.9, 16.2, 16.1, 16.4, 16.4

[3] A. Beauville, Annulation du $H^{1}$ pour les fibrés en droites plats, in: Complex algebraic varieties (Bayreuth, 1990), 1-15, Lecture Notes in Math., vol. 1507, Springer, Berlin, 1992. MR1178716 16.6

[4] M. Bestvina, N. Brady, Morse theory and finiteness properties of groups, Invent. Math. 129 (1997), no. 3, 445-470. MR1465330 1.8, 2.3, 15.2

[5] R. Bieri, Homological dimension of discrete groups, Second edition, Queen Mary Coll. Math. Notes, Queen Mary College, Dept. Pure Math., London, 1981. MR0715779 2.2

[6] R. Bieri, Finiteness length and connectivity length for groups, in: Geometric group theory down under, 9-22, de Gruyter, Berlin, 1999. MR1714837 1.6, 2.1, 13.3

[7] R. Bieri, Deficiency and the geometric invariants of a group, J. Pure Appl. Alg. 208 (2007), no. 3, 951-959. MR2283437 8, 8.2, 8.1, 11.2

[8] R. Bieri, R. Geoghegan, Sigma invariants of direct products of groups, preprint arxiv:0808.0013. 1.6, $13.3,13.4,13.3$

[9] R. Bieri, J. R. J. Groves, The geometry of the set of characters induced by valuations, J. Reine Angew. Math. 347 (1984), 168-195. MR0733052 1.1, 10.1

[10] R. Bieri, W. Neumann, R. Strebel, A geometric invariant of discrete groups, Invent. Math. 90 (1987), no. 3, 451-477. MR0914846 1.1, 1.2, 7, 7.1, 7.1, 7.2, 7.4, 11.2, 13.3

[11] R. Bieri, B. Renz, Valuations on free resolutions and higher geometric invariants of groups, Comment. Math. Helvetici 63 (1988), no. 3, 464-497. MR0960770 1.1, 7, 7.1, 7.2, 7.1, 7.3, 7.4, 11.2

[12] K. S. Brown, Trees, valuations, and the Bieri-Neumann-Strebel invariant, Invent. Math. 90 (1987), no. 3, 479-504. MR0914847 1.1, 11.2

[13] K.-U. Bux, C. Gonzalez, The Bestvina-Brady construction revisited: geometric computation of $\Sigma$ invariants for right-angled Artin groups, J. London Math. Society 60 (1999), no. 3, 793-801. MR1753814 1.7, 14.3, 14.4

[14] D. Cohen, Resonance of basis-conjugating automorphism groups, Proc. Amer. Math. Soc. 137 (2009), no. 9, 2835-2841. MR2506439 11.1

[15] D. Cohen, A. Suciu, Characteristic varieties of arrangements, Math. Proc. Cambridge Phil. Soc. 127 (1999), no. 1, 33-53. MR1692519 13.1

[16] P. Deligne, Théorie de Hodge. II, Inst. Hautes Études Sci. Publ. Math. 40 (1972), 5-57; III, ibid 44 (1974), 5-77. MR0498551, MR0498552. 16.1

[17] P. Deligne, P. Griffiths, J. Morgan, D. Sullivan, Real homotopy theory of Kähler manifolds, Invent. Math. 29 (1975), no. 3, 245-274. MR0382702 1.9, 5.3, 16.1

[18] T. Delzant, Trees, valuations and the Green-Lazarsfeld set, Geom. Funct. Anal. 18 (2008), no. 4, 1236-1250. MR2465689 1.4, 10.2

[19] T. Delzant, L'invariant de Bieri Neumann Strebel des groupes fondamentaux des variétés kählériennes, preprint arxiv:math.DG/0603038, to appear in Math. Annalen. 1.9, 16.4

[20] A. Dimca, L. Maxim, Multivariable Alexander polynomials of hypersurface complements, Trans. Amer. Math. Soc. 359 (2007), no. 7, 3505-3528. MR2299465 3.3 
[21] A. Dimca, S. Papadima, A. Suciu, Alexander polynomials: Essential variables and multiplicities, Int. Math. Res. Notices 2008, no. 3, Art. ID rnm119, 36 pp. MR2416998 3.5

[22] A. Dimca, S. Papadima, A. Suciu, Formality, Alexander invariants, and a question of Serre, preprint arxiv:math.AT/0512480. 1.3, 5.2, 5.5, 13.1, 13.2, 14.2, 16.4

[23] A. Dimca, S. Papadima, A. Suciu, Topology and geometry of cohomology jump loci, Duke Math. Journal 148 (2009), no. 3, 405-457. MR2527322 1.1, 1.6, 1.9, 4.2, 4.3, 4.2, 5.6, 16.2

[24] A. Dimca, A. Suciu, Which 3-manifold groups are Kähler groups?, J. Eur. Math. Soc. 11 (2009), no. 3, 521-528. MR2505439 11.1

[25] W. G. Dwyer, D. Fried, Homology of free abelian covers. I, Bull. London Math. Soc. 19 (1987), no. 4, 350-352. MR0887774 1.2, 1.5, 1.5, 6, 6.1, 6.4, 6.9

[26] H. Esnault, V. Schechtman, E. Viehweg, Cohomology of local systems of the complement of hyperplanes, Invent. Math. 109 (1992), no. 3, 557-561; Erratum, ibid. 112 (1993), no. 2, 447. MR1176205, MR1213111 1.9, 16.5

[27] M. Farber, Topology of closed one-forms, Math. Surveys Monogr., vol. 108, Amer. Math. Soc., Providence, RI, 2004. MR2034601 1.4, 8, 8.1, 8.3, 8.4, 8.4, 9, 10.2, 10.2

[28] M. Farber, R. Geoghegan, D. Schütz, Closed 1-forms in topology and geometric group theory, preprint arxiv:0810.0962 1.1, 8.2

[29] M. Farber, A. Suciu, S. Yuzvinsky, Mini-Workshop: Topology of closed one-forms and cohomology jumping loci, Oberwolfach Reports 4 (2007), no. 3, 2321-2360. MR2432117 1.4

[30] R. H. Fox, Free differential calculus. I. Derivation in the free group ring, Ann. of Math. 57 (1953), 547-560. MR0053938 3.2, 3.5

[31] R. Gehrke, The higher geometric invariants for groups with sufficient commutativity, Comm. Algebra 26 (1998), no. 4, 1097-1115. MR1612192 13.3

[32] E. Hironaka, Alexander stratifications of character varieties, Ann. Inst. Fourier (Grenoble) 47 (1997), no. 2, 555-583. MR1450425 13.1, 13.2

[33] M. Kapovich, J. Millson, On representation varieties of Artin groups, projective arrangements and the fundamental groups of smooth complex algebraic varieties, Inst. Hautes Études Sci. Publ. Math. 88 (1998), no. 8, 5-95. MR1733326 5.3

[34] A. Libgober, First order deformations for rank one local systems with a non-vanishing cohomology, Topology Appl. 118 (2002), no. 1-2, 159-168. MR1877722 1.3, 5.5

[35] A. Macinic, Cohomology rings and formality properties of nilpotent groups, preprint arxiv:0801.4847 13.1

[36] A. Macinic, S. Papadima, Characteristic varieties of nilpotent groups and applications, in: Proceedings of the Sixth Congress of Romanian Mathematicians (Bucharest, 2007), vol. 1, 57-64, Romanian Academy, Bucharest, 2009. 12.2, 12.2

[37] D. Matei, A. Suciu, Hall invariants, homology of subgroups, and characteristic varieties, Internat. Math. Res. Notices 2002 (2002), no. 9, 465-503. MR1884468 1.3, 5.5

[38] J. Meier, Geometric invariants for Artin groups, Proc. London Math. Soc. (3) 74 (1997), no. 1, 151-173. MR1416729 13.3

[39] J. Meier, H. Meinert, L. VanWyk, Higher generation subgroup sets and the $\Sigma$-invariants of graph groups, Comment. Math. Helv. 73 (1998), no. 1, 22-44. MR1610579 1.1, 1.7, 14.3, 14.4, 15.11

[40] J. W. Morgan, The algebraic topology of smooth algebraic varieties, Inst. Hautes Études Sci. Publ. Math. 48 (1978), 137-204. MR0516917 16.1, 16.3

[41] A. Pajitnov, On the sharpness of inequalities of Novikov type for manifolds with a free abelian fundamental group, Math. USSR-Sb. 68 (1991), no. 2, 351-389. MR1034426 8.3

[42] A. Pajitnov, Novikov homology, twisted Alexander polynomials, and Thurston cones, St. Petersburg Math. J. 18 (2007), no. 5, 809-835 MR2301045 1.4

[43] S. Papadima, A. Suciu, Chen Lie algebras, Int. Math. Res. Notices 2004, no. 21, 1057-1086. MR2037049 5.2, 5.3

[44] S. Papadima, A. Suciu, Algebraic invariants for right-angled Artin groups, Math. Annalen 334 (2006), no. 3, 533-555. MR2207874 1.7, 13.2, 14.2, 14.3

[45] S. Papadima, A. Suciu, Algebraic invariants for Bestvina-Brady groups, J. London Math. Society, 76 (2007), no. 2, 273-292. MR2363416 5.3, 15.2, 15.3 
[46] S. Papadima, A. Suciu, Toric complexes and Artin kernels, Advances in Math. 220 (2009), no. 2, 441-477. MR2466422 1.7, 1.8, 6.3, 14.1, 14.2, 14.2, 15.2, 15.5, 15.2, 15.3, 15.4, 15.11

[47] S. Papadima, A. Suciu, Algebraic monodromy and obstructions to formality, preprint arxiv:0901.0105, to appear in Forum Math. (2010). 11.1

[48] D. Quillen, Rational homotopy theory, Ann. of Math. 90 (1969), 205-295. MR0258031 5.3

[49] D. Schütz, On the direct product conjecture for sigma invariants, Bull. London Math. Soc. 40 (2008), no. 4, 675-684. MR2441140 13.3

[50] J.-Cl. Sikorav, Homologie de Novikov associée à une classe de cohomologie réele de degré un, Thèse Orsay, 1987. 1.1, 8, 8.2

[51] J. Stallings, On fibering certain 3-manifolds, in: Topology of 3-manifolds and related topics, pp. 95-100, Prentice-Hall, Englewood Cliffs, N.J., 1962. MR0158375 1.2, 7.3

[52] J. Stallings, A finitely presented group whose 3-dimensional integral homology is not finitely generated, Amer. J. Math. 85 (1963), 541-543. MR0158917 1.2, 1.8

[53] D. Sullivan, Infinitesimal computations in topology, Inst. Hautes Études Sci. Publ. Math. 47 (1977), 269-331. MR0646078 5.3

[54] W.P. Thurston, A norm for the homology of 3-manifolds, Mem. Amer. Math. Soc., 59 (1986), no. 339. MR0823443 1.2

Institute of Mathematics Simion Stoilow, P.O. Box 1-764, RO-014700 Bucharest, Romania E-mail address: Stefan.Papadima@imar.ro

Department of Mathematics, Northeastern University, Boston, MA 02115, USA

E-mail address: a.suciu@neu.edu 